Pacific

Journal of

Mathematics

\title{
UNITARY PRINCIPAL SERIES OF SPLIT ORTHOGONAL GROUPS
}

Alessandra Pantano, Annegret Paul and Susana Salamanca Riba 


\title{
UNITARY PRINCIPAL SERIES OF SPLIT ORTHOGONAL GROUPS
}

\author{
Alessandra Pantano, Annegret Paul and Susana Salamanca Riba
}

\begin{abstract}
We prove the nonunitarity of a large set of parameters for Langlands quotients of minimal principal series of the orthogonal group $\operatorname{SO}(n+1, n)$, by showing that the set of unitary principal series parameters of $\operatorname{SO}(n+1, n)$ embeds into a (known) union of spherical unitary parameters for certain split orthogonal groups. In an earlier paper, we proved the nonunitarity of the genuine principal series of the metaplectic group $\operatorname{Mp}(2 n)$ attached to the same set of parameters. We conjecture that the set of parameters is complete in both cases and prove the conjecture for small rank groups and in the case of unipotent parameters.
\end{abstract}

\section{Introduction}

For $G=\mathrm{SO}(n+1, n)$ or the real metaplectic group $\mathrm{Mp}(2 n)$, let $M A$ be the Levi factor of a minimal parabolic subgroup of $G$. For every irreducible representation $\delta$ of $M$ and every real character $v$ of $A$, we choose a minimal parabolic subgroup $P=M A N$ of $G$ making $v$ weakly dominant, and we denote by

$$
I_{G}(\delta, v):=\operatorname{Ind}_{P}^{G}(\delta \otimes v)
$$

the (minimal) principal series representation of $G$ induced from the representation $\delta \otimes v \otimes$ triv of $P$. In the case of the metaplectic group, we assume that the representation is genuine, that is, does not factor to the symplectic group. Let $J_{G}(\delta, v)$ be the Langlands quotient of $I_{G}(\delta, v)$, that is, the distinguished irreducible composition factor containing the minimal $K$-type. We are interested in determining for which pairs $(\delta, v)$ the irreducible representation $J_{G}(\delta, v)$ is unitarizable. We call this set the complementary series $\operatorname{CS}(G)$ of $G$. The spherical complementary series of $\mathrm{SO}(n+1, n)_{0}$ (with $\delta$ trivial) is denoted $\mathrm{CS}\left(\mathrm{SO}(n+1, n)_{0}, \delta_{0}\right)$. Our work is motivated by the following conjecture.

This material is based on work supported by NSF Grants DMS-0554278, DMS-0967583, and DMS0967168.

MSC2010: 22E45.

Keywords: orthogonal groups, intertwining operators, petite K-types, complementary series, theta correspondence, unipotent representations, spherical unitary dual, Weyl group representations. 
Conjecture 1. There is a natural, well-defined bijection

$$
\mathrm{CS}(G) \longleftrightarrow \bigcup_{p+q=n} \mathrm{CS}\left(\mathrm{SO}(p+1, p)_{0}, \delta_{0}\right) \times \mathrm{CS}\left(\mathrm{SO}(q+1, q)_{0}, \delta_{0}\right)
$$

Because the spherical unitary dual of every split orthogonal group is known, by [Barbasch 2010], this would give a complete description of the unitary principal series for these two families of groups $G$.

For the two groups under consideration, $A$ is isomorphic to $\mathbb{R}^{n}$, and $M$ is isomorphic to $(\mathbb{Z} / 2 \mathbb{Z})^{n}$, in the case of $\mathrm{SO}(n+1, n)$, and to $\mathbb{Z} / 4 \mathbb{Z} \times(\mathbb{Z} / 2 \mathbb{Z})^{n-1}$ for $\operatorname{Mp}(2 n)$; moreover there is a natural one-to-one correspondence between $M$ types (i.e., irreducible representations of $M)$ of $\mathrm{SO}(n+1, n)$ and genuine $M$-types of $\mathrm{Mp}(2 n)$. Every $M$-type of $\mathrm{SO}(n+1, n)$ and every genuine $M$-type of $\mathrm{Mp}(2 n)$ is contained in a unique fine $K$-type for $G$. Fix an $M$-type $\delta$. We call the set of real parameters $v$ for which $J_{G}(\delta, v)$ is unitary the $\delta$-complementary series of $G$ :

$$
\mathrm{CS}(G, \delta):=\left\{v \in \mathfrak{a}_{\mathbb{R}}^{*} \mid J_{G}(\delta, v) \text { is unitary }\right\} .
$$

For all $w \in W, J_{G}(\delta, v) \simeq J_{G}(w \delta, w v)$; hence

$$
\operatorname{CS}(G, w \cdot \delta)=w^{-1} \cdot \operatorname{CS}(G, \delta) .
$$

It follows that $\operatorname{CS}(G, \delta)$ is invariant under the action of the stabilizer $W^{\delta}$ of $\delta$ in $W$ and depends only on the orbit of the $M$-type $\delta$ under the action of $W$. Here $W$ is the Weyl group of $A$ in $G$, which may be identified with the Weyl group of the root system of $G$.

The Weyl groups of $\mathrm{SO}(n+1, n)$ and of $\mathrm{Mp}(2 n)$ are isomorphic. Moreover if an $M$-type of $\mathrm{SO}(n+1, n)$ and a genuine $M$-type of $\mathrm{Mp}(2 n)$ correspond to each other in the above mentioned bijection, then their stabilizers are also isomorphic. The $W$-orbits of $M$-types of $\mathrm{SO}(n+1, n)$ and of genuine $M$-types of $\mathrm{Mp}(2 n)$ are parametrized by pairs of nonnegative integers $(p, q)$ with $p+q=n$; we choose a representative $\delta^{p, q}$ in each orbit (see (2-15)). Then the $W^{\delta^{p, q}}$-action leads to a natural splitting of each real parameter $v$ into a pair $\left(v^{p}, v^{q}\right) \in \mathbb{R}^{p} \times \mathbb{R}^{q}$ (see Section 3).

In [Pantano et al. 2010], we proved that the $\delta^{p, q}$-complementary series of $\mathrm{Mp}(2 n)$ embeds into the product of the spherical complementary series of $\mathrm{SO}(p+1, p)_{0}$ with that of $\mathrm{SO}(q+1, q)_{0}$. In this paper, we show the analogous result for $\mathrm{SO}(n+1, n)$. In particular, the following theorem and the corresponding result for $\operatorname{Mp}(2 n)$ make Conjecture 1 more precise.

Theorem 2. Let $G=\mathrm{SO}(n+1, n)$, and let $v=\left(v_{1}, \ldots, v_{n}\right)$ be a real character of $A$. For each pair of nonnegative integers $(p, q)$ such that $p+q=n$, write $v=\left(v^{p} \mid v^{q}\right)$ with 


$$
v^{p}:=\left(v_{1}, \ldots, v_{p}\right) \quad \text { and } \quad v^{q}:=\left(v_{p+1}, \ldots, v_{n}\right)
$$

The map

$$
\mathrm{CS}\left(G, \delta^{p, q}\right) \rightarrow \mathrm{CS}\left(\mathrm{SO}(p+1, p)_{0}, \delta_{0}\right) \times \mathrm{CS}\left(\mathrm{SO}(q+1, q)_{0}, \delta_{0}\right)
$$

taking $v$ to $\left(v^{p}, v^{q}\right)$ is a well-defined injection. Here $\delta_{0}$ denotes the trivial M-type.

The theorem asserts that if the (Hermitian) Langlands quotient $J\left(\delta^{p, q},\left(v^{p} \mid v^{q}\right)\right)$ of $G$ is unitary, then the spherical (Hermitian) Langlands quotients $J\left(\delta_{0}, v^{p}\right)$ of $\mathrm{SO}(p+1, p)_{0}$ and $J\left(\delta_{0}, v^{q}\right)$ of $\mathrm{SO}(q+1, q)_{0}$ must both be unitary. Due to [Barbasch 2010], these conditions can be explicitly checked. We give a description of the spherical unitary parameters for $\mathrm{SO}(p+1, p)_{0}$ in Section 8 .

Corollary 3. Let $G=\mathrm{SO}(n+1, n)$ and let $v=\left(v^{p} \mid v^{q}\right)$ be a real character of $A$, as in (1-4). If the spherical Langlands quotient $J\left(\delta_{0}, v^{p}\right)$ of $\mathrm{SO}(p+1, p)_{0}$ or the spherical Langlands quotient $J\left(\delta_{0}, v^{q}\right)$ of $\mathrm{SO}(q+1, q)_{0}$ is not unitary, then the Langlands quotient $J\left(\delta^{p, q},\left(v^{p} \mid v^{q}\right)\right)$ of $\mathrm{SO}(n+1, n)$ is also not unitary.

The theorem gives nonunitarity certificates for $\mathrm{SO}(n+1, n)$. In general, proving the unitarity of a representation is much harder than showing that it is not unitary. We conjecture that the spherical complementary series of $\mathrm{SO}(p+1, p)_{0} \times \mathrm{SO}(q+1, q)_{0}$ gives an exhaustive parametrization of unitary parameters for the Langlands quotients of the $\delta^{p, q}$-principal series of both $G=\mathrm{Mp}(2 n)$ and $\mathrm{SO}(n+1, n)$. To prove this we must show that, for each pair of parameters

$$
v^{p} \in \mathrm{CS}\left(\mathrm{SO}(p+1, p)_{0}, \delta_{0}\right) \quad \text { and } \quad v^{q} \in \operatorname{CS}\left(\operatorname{SO}(q+1, q)_{0}, \delta_{0}\right),
$$

the Langlands quotient $J_{G}\left(\delta^{p, q},\left(v^{p} \mid v^{q}\right)\right)$ of $G$ is unitary for both $G=\operatorname{Mp}(2 n)$ and $\mathrm{SO}(n+1, n)$. In this paper, we show that it is sufficient to prove this for one of the two families of groups.

Theorem 4. Suppose the Langlands quotient $J_{G}\left(\delta^{p, q},\left(v^{p} \mid v^{q}\right)\right)$ is unitary for all $v^{p} \in \mathrm{CS}\left(\mathrm{SO}(p+1, p)_{0}, \delta_{0}\right)$ and $\nu^{q} \in \mathrm{CS}\left(\operatorname{SO}(q+1, q)_{0}, \delta_{0}\right)$, and all $p+q=n$, for $G=\mathrm{Mp}(2 n)$. Then the same is true for $G=\mathrm{SO}(n+1, n)$; and vice versa.

In [Pantano et al. 2010], we proved the unitarity of the principal series of $\operatorname{Mp}(2 n)$ attached to our list of parameters for some small rank cases; for the general case, we exhibited two large families of spherical unitary parameters for the product $\mathrm{SO}(p+1, p)_{0} \times \mathrm{SO}(q+1, q)_{0}$, which give rise to $\delta^{p, q}$-complementary series of $\mathrm{Mp}(2 n)$. In this paper, we show some of the $\mathrm{SO}(n+1, n)$ analogues of these results. In particular, we obtain:

Theorem 5. Let $n=p+q \leq 4$ and take elements $v^{p} \in \mathrm{CS}\left(\operatorname{SO}(p+1, p)_{0}, \delta_{0}\right)$ and $\nu^{q} \in \operatorname{CS}\left(\operatorname{SO}(q+1, q)_{0}, \delta_{0}\right)$. Then $J_{G}\left(\delta^{p, q},\left(\nu^{p} \mid v^{q}\right)\right)$ of $G$ is unitary for both $G=\mathrm{Mp}(2 n)$ and $\mathrm{SO}(n+1, n)$. 
Proving the unitarity of the $\delta$-principal series for our collection of parameters might use the ideas for Barbasch's proof [2010] in the spherical case. First using normalized parabolic induction and deformation of parameters, Barbasch reduces the question of unitarity to unipotent parameters. These are parameters that correspond to special unipotent representations, that is, those that are attached to unipotent Arthur parameters [1989]. Then he proves the unitarity of these special unipotent representations.

Arthur packets and "unipotent" parameters are defined for linear groups only; for genuine $(p, q)$-principal series representations of the metaplectic group, we call a parameter $v=\left(v^{p} \mid v^{q}\right)$ unipotent if it is a unipotent parameter for $\operatorname{SO}(n+1, n)$.

In his recent book [Arthur 2013], the author proves that for split classical groups, all special unipotent representations are unitary. We check that $J_{\mathrm{SO}(n+1, n)}\left(\delta^{p, q}, v\right)$ is special unipotent if and only if $v^{p}$ and $v^{q}$ are spherical unipotent parameters for $\mathrm{SO}(p+1, p)_{0}$ and $\mathrm{SO}(q+1, q)_{0}$, respectively. Using the theta correspondence for dual pairs of the form $(O(r, s), \operatorname{Sp}(2 m, \mathbb{R}))$, we prove that the unitarity of the special unipotent principal series of $\mathrm{SO}(n+1, n)$ implies the unitarity of the corresponding representation of $\operatorname{Mp}(2 n)$. We obtain the following result.

Theorem 6. If $v^{p}, v^{q}$ are spherical unipotent parameters for $\mathrm{SO}(p+1, p)_{0}$ and $\mathrm{SO}(q+1, q)_{0}$, respectively, then $v=\left(v^{p} \mid v^{q}\right) \in \mathrm{CS}\left(G, \delta^{p, q}\right)$ for $G=\operatorname{Mp}(2 n)$ and $\mathrm{SO}(n+1, n)$.

Sections 2 through 4 of this paper contain the proof of Theorem 2. In Section 2, we collect some structural facts about our groups and the $K$-types. Section 3 contains a careful outline of the main argument. Here we reduce the proof of our main theorem to an explicit matching of $W$-types (Theorem 9). The heart of the calculations is in Section 4, with the proof of Theorem 9. In Sections 5 through 7, we address what we know about the unitarity of the $\delta$-principal series. Section 5 is devoted to proving Theorem 5. In Section 6, we use the theta correspondence to relate the complementary series of $\mathrm{SO}(n+1, n)$ and $\mathrm{Mp}(2 n)$ to each other. Unipotent parameters and their unitarity are discussed in Section 7, and in Section 8, we give a description of the spherical unitary parameters for $\mathrm{SO}(n+1, n)$.

\section{The structure of $\operatorname{SO}(n+1, n)$}

Let $G=\mathrm{SO}(n+1, n)$ be defined by

$$
G=\operatorname{SO}(n+1, n):=\left\{g \in \operatorname{SL}(2 n+1, \mathbb{R}):\left(g^{t}\right) J g=J\right\},
$$

with $J=\operatorname{diag}\left(-I_{n+1}, I_{n}\right)$. Here $I_{s}$ denotes the identity matrix of size $s \times s$. We denote by $\mathfrak{g}_{0}$ the Lie algebra of $G$, and by $\mathfrak{g}$ its complexification. Let $\mathfrak{k}_{0}$ be the maximal compact Cartan subalgebra of $\mathfrak{g}_{0}$ corresponding to the Cartan involution $\theta(X)=-X^{t}$, and let $K=S(O(n+1) \times O(n))$ be the corresponding compact 
subgroup of $G$. Write $\mathfrak{g}_{0}=\mathfrak{k}_{0} \oplus \mathfrak{p}_{0}$ for the Cartan decomposition of $\mathfrak{g}_{0}$, and let $\mathfrak{a}_{0}$ be the maximal abelian subspace

$$
\mathfrak{a}_{0}=\left\{X(B):=\left(\begin{array}{cc}
0 & B \\
B^{t} & 0
\end{array}\right): B=\left(\begin{array}{ccccc}
0 & 0 & \ldots & 0 & 0 \\
0 & 0 & \ldots & 0 & s_{n} \\
0 & 0 & \ldots & s_{n-1} & 0 \\
\vdots & \vdots & \ddots & \vdots & \vdots \\
0 & s_{2} & \ldots & 0 & 0 \\
s_{1} & 0 & \ldots & 0 & 0
\end{array}\right), s_{1}, \ldots, s_{n} \in \mathbb{R}\right\}
$$

of $\mathfrak{p}_{0}$. For all $i=1, \ldots, n$, let $\epsilon_{i} \in \mathfrak{a}_{0}^{*}$ be defined by $\epsilon_{i}(X(B))=s_{i}$. Then the restricted roots are

$$
\Delta\left(\mathfrak{g}_{0}, \mathfrak{a}_{0}\right)=\left\{ \pm \epsilon_{i} \pm \epsilon_{j}\right\}_{1 \leq i<j \leq n} \cup\left\{ \pm \epsilon_{k}\right\}_{k=1, \ldots, n} .
$$

They form a root system $\Delta$ of type $B_{n}$. The corresponding Weyl group $W=W(\Delta)$ is isomorphic to $S_{n} \ltimes(\mathbb{Z} / 2 \mathbb{Z})^{n}$, and consists of all permutations and sign changes on $n$ coordinates. Note that $W$ can be realized as $N_{K}(A) / Z_{K}(A)$, where $A$ is the vector group $\exp \left(\mathfrak{a}_{0}\right)$.

For each root $\alpha \in \Delta$ we choose a Lie algebra homomorphism

$$
\phi_{\alpha}: \mathfrak{s l}(2, \mathbb{R}) \rightarrow \mathfrak{g}_{0}=\mathfrak{s o}(n+1, n),
$$

as in [Vogan 1981, (4.3.6)], and we let $G_{\alpha}$ be the corresponding connected subgroup of $\mathrm{SO}(n+1, n)$. Moreover we define

$$
\begin{aligned}
Z_{\alpha} & :=\phi_{\alpha}\left(\begin{array}{rr}
0 & 1 \\
-1 & 0
\end{array}\right), \\
\sigma_{\alpha} & :=\exp \left(\frac{\pi}{2} Z_{\alpha}\right), \\
m_{\alpha} & :=\exp \left(\pi Z_{\alpha}\right)=\sigma_{\alpha}^{2} .
\end{aligned}
$$

We make the following choices:

$$
\begin{aligned}
Z_{\epsilon_{i}+\epsilon_{j}} & =\left(E_{n+2-j, n+2-i}-E_{n+2-i, n+2-j}\right)+\left(E_{n+1+i, n+1+j}-E_{n+1+j, n+1+i}\right), \\
Z_{\epsilon_{i}-\epsilon_{j}} & =\left(E_{n+2-j, n+2-i}-E_{n+2-i, n+2-j}\right)-\left(E_{n+1+i, n+1+j}-E_{n+1+j, n+1+i}\right) \\
Z_{\epsilon_{k}} & =2 E_{1, n+2-k}-2 E_{n+2-k, 1} .
\end{aligned}
$$

Then

$$
\sigma_{\epsilon_{k}}=I-2\left(E_{1,1}+E_{n+2-k, n+2-k}\right), \quad m_{\epsilon_{k}}=I,
$$

and for $\alpha=\epsilon_{i} \pm \epsilon_{j}$,

$$
\begin{aligned}
\sigma_{\alpha} & =I-\left(E_{n+2-i, n+2-i}+E_{n+2-j, n+2-j}+E_{n+1+i, n+1+i}+E_{n+1+j, n+1+j}\right)+Z_{\alpha}, \\
m_{\alpha} & =I-2\left(E_{n+2-i, n+2-i}+E_{n+2-j, n+2-j}+E_{n+1+i, n+1+i}+E_{n+1+j, n+1+j}\right) .
\end{aligned}
$$


As usual, the symbol $E_{i, j}$ denotes the $(i, j)$-elementary matrix.

The centralizer of $A$ in $K$ is denoted by $M$, and consists of all elements

$$
T\left(t_{1}, \ldots, t_{n}\right)=\operatorname{diag}\left(1 ; t_{n}, t_{n-1}, \ldots, t_{1} ; t_{1}, t_{2}, \ldots, t_{n}\right),
$$

with $t_{1}, \ldots, t_{n}= \pm 1$. It is an abelian group of order $2^{n}$, isomorphic to $(\mathbb{Z} / 2 \mathbb{Z})^{n}$. The dual group $\widehat{M}$ is generated by the $M$-types $\left\{\delta_{i}: 1 \leq i \leq n\right\}$, where

$$
\delta_{i}\left(T\left(t_{1}, \ldots, t_{n}\right)\right):=t_{i} .
$$

For every subset $S$ of $\{1,2, \ldots, n\}$, we denote by $\delta_{S}$ the irreducible representation of $M$ satisfying

$$
\delta_{S}\left(T\left(t_{1}, \ldots, t_{n}\right)\right)=\prod_{i \in S} t_{i}
$$

The Weyl group $W$ acts on the set of irreducible representations of $M$ by

$$
\left(s_{\alpha} \cdot \delta\right)(m):=\delta\left(\sigma_{\alpha}^{-1} m \sigma_{\alpha}\right) \text { for all } m \in M \text { and } \alpha \in \Delta .
$$

The stabilizer of $\delta$ in $W$ is the subgroup

$$
W^{\delta}:=\{w \in W: w \cdot \delta \simeq \delta\}
$$

of $W$. It is easy to check that

$$
s_{\epsilon_{k}} \cdot \delta_{S}=\delta_{S} \quad \text { and } \quad s_{\epsilon_{i} \pm \epsilon_{j}} \cdot \delta_{S}= \begin{cases}\delta_{S} & \text { if } i, j \in S \text { or } i, j \in S^{C}, \\ \delta_{S \triangle\{i, j\}} & \text { otherwise, }\end{cases}
$$

for all $1 \leq i<j \leq n$ and all $k=1, \ldots, n$. Here $S^{C}$ denotes the complement of $S$ in $\{1,2, \ldots, n\}$, and the symbol $(S \triangle T)$ denotes the symmetric difference of the two subsets. If $q=\# S$ (the cardinality of $S$ ) and $p=\#\left(S^{C}\right)$, this is the Weyl group of a root system of type $B_{p} \times B_{q}$.

Equation (2-13) also shows that

$$
W \cdot \delta_{S}=\left\{\delta_{T}: \# S=\# T\right\} .
$$

This implies that the Weyl group orbits of $\widehat{M}$ can be parametrized by pairs of nonnegative integers $(p, q)$ with $p+q=n$; for each such pair, we choose a representative

$$
\delta^{p, q}:=\delta_{\{p+1, p+2, \ldots, n\}}=\delta_{p+1} \cdot \delta_{p+2} \cdots \delta_{n} .
$$

With this notation, $W^{\delta^{p, q}}=W\left(B_{p}\right) \times W\left(B_{q}\right)$, and the trivial $M$-type is $\delta^{n, 0}$.

Let $\delta$ be an irreducible representation of $M$. A root $\alpha \in \Delta$ is called "good" for $\delta$ if $\delta\left(m_{\alpha}\right) \neq-1$. Otherwise we say that $\alpha$ is a "bad $\operatorname{root"}$ for $\delta$. The set of $\operatorname{good}$ roots for $\delta=\delta_{S}$ is

$$
\Delta_{\delta_{S}}=\left\{ \pm \epsilon_{i} \pm \epsilon_{j}: i, j \in S \text { or } i, j \in S^{C}\right\} \cup\left\{ \pm \epsilon_{k}: 1 \leq k \leq n\right\} .
$$


If $q=\# S$ and $p=\#\left(S^{C}\right)$, this is a root system of type $B_{p} \times B_{q}$.

Remark 7. For every $M$-type $\delta$, the Weyl group of the root system $\Delta_{\delta}$ coincides with the stabilizer $W^{\delta}$ of $\delta$ in $W$.

Recall the definition of fine $K$-types given in [Vogan 1981, Section 4] (see also [Adams et al. 2007, Definition 4.9]). The fine $K$-types of $\mathrm{SO}(n+1, n)$ are given by

$$
\mu_{p, q}=\operatorname{triv} \otimes \Lambda^{q}\left(\mathbb{C}^{n}\right)
$$

for each value of $0 \leq q \leq n$. The restriction of $\mu_{p, q}$ to $M$ is

$$
W \cdot \delta^{p, q}
$$

(see (2-15)), and its highest weight is

$$
\begin{cases}(0, \ldots, 0 ; \underbrace{1, \ldots, 1}_{q}, 0, \ldots, 0 ;+) & \text { if } q \leq n / 2, \\ (0, \ldots, 0 ; \underbrace{1, \ldots, 1}_{n-q}, 0, \ldots, 0 ;-) & \text { otherwise. }\end{cases}
$$

Recall that since $K=S(O(n+1) \times O(n))$ is disconnected, the highest weight does not necessarily determine the $K$-type uniquely, so we use a sign to distinguish two representations with the same highest weight.

Remark 8. (a) The restriction of a fine $K$-type to $M$ consists of the $W$-orbit of a single $M$-type.

(b) Every $M$-type $\delta$ is contained in the restriction to $M$ of a unique fine $K$-type $\mu_{\delta}$.

\section{Nonunitarity certificates for complementary series of $\operatorname{SO}(n+1, n)$}

For $G=\mathrm{SO}(n+1, n)$, recall the definition of the $\delta$-complementary series $\operatorname{CS}(G, \delta)$ in (1-2). This is a closed set because unitarity is a closed condition. As seen in Section 2, it suffices to consider the complementary series attached to a single $M$-type in each $W$-orbit for the action of the Weyl group on $\widehat{M}$. Such orbits are parametrized by pairs of nonnegative integers $(p, q)$ with $p+q=n$. In each orbit, we choose the representative $\delta^{p, q}$ introduced in (2-15); the corresponding fine $K$-type is $\mu_{\delta^{p, q}}$ (see (2-17)). The stabilizer $W^{\delta^{p, q}} \cong W\left(B_{p}\right) \times W\left(B_{q}\right)$ acts on a (real) continuous parameter $v \in \mathfrak{a}_{\mathbb{R}}^{*}$ by sign changes and separate permutations of the first $p$ and the last $q$ coordinates. This leads to a natural splitting of each parameter $v$ into a pair $\left(v^{p} \mid v^{q}\right)$ as in Theorem 2 .

Theorem 2 gives a comparison between the set of (real) unitary parameters for principal series representations of different groups. On the one hand, we have a $\delta^{p, q}$-principal series of the group $G=\mathrm{SO}(n+1, n)$; on the other hand, we have a 
spherical principal series for the group

$$
G^{\delta^{p, q}}=\mathrm{SO}(p+1, p)_{0} \times \mathrm{SO}(q+1, q)_{0} .
$$

This group is intrinsically related to the $M$-type $\delta^{p, q}$ of $G$, and only depends on the system of good roots $\Delta_{\delta, q}$ (see (2-16)) associated to this $M$-type. Precisely, $G^{\delta^{p, q}}$ is the connected real split group corresponding to the root system

$$
\Delta\left(G^{\delta^{p, q}}\right):=\Delta_{\delta^{p, q}} .
$$

Consider a parameter $v=\left(v^{p} \mid v^{q}\right)$ as in Theorem 2. Write $I_{G^{\delta}}{ }^{p}\left(\delta_{0}, v\right)$ for the spherical principal series of $G^{\delta^{p, q}}$ with parameter $v$, and consider the possibly nonspherical representation $I\left(\delta^{p, q}, v\right)$ of $\mathrm{SO}(n+1, n)$ with the same parameter. The long Weyl group element of both $G$ and $G^{\delta^{p, q}}$ is equal to - Id, hence

$$
w_{0} \cdot v=-v, \quad w_{0} \cdot \delta_{0}=\delta_{0}, \quad w_{0} \cdot \delta^{p, q}=\delta^{p, q} .
$$

These are exactly the conditions that $v, \delta_{0}$ and $\delta^{p, q}$ must satisfy so that the above mentioned principal series representations admit an invariant Hermitian form. If $v$ is (weakly) dominant, then the Langlands quotients $J_{G^{\delta, q}}\left(\delta_{0}, v\right)$ and $J\left(\delta^{p, q}, v\right)$ are the quotients of the appropriate principal series by the radical of the Hermitian form. Hence they inherit a nondegenerate Hermitian form, and they are unitary if and only if the original form on the principal series is (positive) semidefinite.

To study the unitarity of a Langlands quotient, one needs to look at the signature of the Hermitian intertwining operators on the principal series which induce the form. Luckily these intertwining operators are very well understood. A thorough description can be found in [Barbasch et al. 2008] for split linear groups (such as $\mathrm{SO}(n+1, n)$ and $\left.G^{\delta^{p, q}}\right)$. We will not review the theory here but only recall the main results. The interested reader may consult the reference above for details.

First consider the spherical Langlands quotient $J_{G^{\delta}, q}\left(\delta_{0}, v\right)$ of the group $G^{\delta^{p, q}}$. Hecke algebra considerations reduce the study of the unitarity of $J_{G^{8}, q}\left(\delta_{0}, v\right)$ to the analysis of the signature of certain (relatively simple) "algebraic" operators. Precisely there is one operator $A\left(w_{0}, \psi, v\right)$ for every representation $\psi$ of the Weyl group of $G^{\delta^{p, q}}$; the representation $J_{G^{\delta, q}}\left(\delta_{0}, v\right)$ is unitary if and only if

$$
A\left(w_{0}, \psi, v\right) \text { is positive semidefinite, for all } \psi \in \widehat{W\left(G^{\delta^{p, q}}\right)} \text {. }
$$

Barbasch [2010] has identified a small set of $W\left(G^{\delta^{p, q}}\right)$-types (called "relevant") that detect unitarity, in the sense that $J_{G^{\delta p q}}\left(\delta_{0}, v\right)$ is unitary if and only if

$A\left(w_{0}, \psi, v\right)$ is positive semidefinite, for all relevant $\psi \in \widehat{W\left(G^{\delta^{p, q}}\right)}$. 
Next we consider the Langlands quotient $J\left(\delta^{p, q}, v\right)$ of $\mathrm{SO}(n+1, n)$. For every $K$-type $\mu$, there is a family of (much harder) "analytic" intertwining operators

$$
T\left(w_{0}, \mu, \delta^{p, q}, v\right),
$$

with the property that the Langlands quotient $J\left(\delta^{p, q}, v\right)$ is unitary if and only if

$$
T\left(w_{0}, \mu, \delta^{p, q}, v\right) \text { is positive semidefinite, for all } \mu \in \widehat{K} \text {. }
$$

The operator $T\left(w_{0}, \mu, \delta^{p, q}, v\right)$ is defined on the $\operatorname{space} \operatorname{Hom}_{K}\left(\mu, I_{G}\left(\delta^{p, q}, v\right)\right)$, which is isomorphic to

$$
V_{\mu}\left[\delta^{p, q}\right]:=\operatorname{Hom}_{M}\left(\mu, \delta^{p, q}\right)
$$

by Frobenius reciprocity. The stabilizer $W^{\delta^{p, q}}$ of the $M$-type $\delta^{p, q}$ acts naturally on this space. Note that the group $W^{\delta^{p, q}}$ coincides with the Weyl group of the system $\Delta_{\delta^{p, q}}$ of good roots for $\delta^{p, q}$ :

$$
W\left(\Delta_{\delta^{p, q}}\right) \simeq W\left(B_{p}\right) \times W\left(B_{q}\right) \simeq W\left(G^{\delta^{p, q}}\right) .
$$

Hence, for every $K$-type $\mu$, we obtain a representation $\psi_{\mu}$ of the Weyl group of $G^{\delta^{p, q}}$ on the domain $V_{\mu}\left[\delta^{p, q}\right]$ of the intertwining operator $T\left(w_{0}, \mu, \delta^{p, q}, v\right)$.

The operator $T\left(w_{0}, \mu, \delta^{p, q}, v\right)$ is in general hard to compute, but if the $K$ type $\mu$ is sufficiently small (more precisely, "petite"; see [Barbasch et al. 2008, Sections 4.5 and 4.6] for a precise definition), then $T\left(w_{0}, \mu, \delta^{p, q}, v\right)$ depends only on the $W\left(\Delta_{\delta^{p, q}}\right)$-structure of $V_{\mu}\left[\delta^{p, q}\right]$. One measure of the size of a $K$-type is its level (see Definition 11).

The following facts are crucial:

(1) Every $K$-type of level at most 2 is automatically petite.

(2) If $\mu$ is petite, then the "analytic" operator on $\mu$ coincides with the "algebraic" operator on the $W\left(\Delta_{\delta^{p, q}}\right)$-type $V_{\mu}\left[\delta^{p, q}\right]$ :

$$
T\left(w_{0}, \mu, \delta^{p, q}, v\right)=A\left(w_{0}, V_{\mu}\left[\delta^{p, q}\right], \delta_{0}, \nu\right) .
$$

(3) For $G=\mathrm{SO}(n+1, n)$, every relevant $W\left(\Delta_{\delta^{p, q}}\right)$-type $\psi$ occurs in the representation of $W\left(\Delta_{\delta^{p, q}}\right)$ on the space $V_{\mu}\left[\delta^{p, q}\right]$ for some petite $K$-type $\mu$ (of level 2).

The first two claims are well known; the proof already appears in [Barbasch et al. 2008] (see also [Pantano et al. 2010] for the corresponding results for double covers of split groups such as $\mathrm{Mp}(2 n))$. The third claim is Theorem 9 below.

Theorem 9. For every relevant $W^{\delta^{p, q}}$-type $\psi$, there exists a $K$-type $\mu$ of level at most 2 such that

$$
\psi_{\mu} \cong \psi
$$


The proof of Theorem 9 is given in Section 4. This concludes the proof of Theorem 2.

Because the spherical unitary dual of split groups of type $B$ is known, Theorem 2 and Corollary 3 provide a set of nonunitarity certificates for (Langlands quotients of) minimal principal series of $\mathrm{SO}(n+1, n)$. We give an example.

By [Adams et al. 2007, Lemma 14.6], the spherical Langlands quotient of $O(k+1, k)$, with parameter, $\left(a_{1} \geq a_{2} \geq \cdots \geq a_{k}\right)$ is not unitary if the last coordinate $a_{k}$ is strictly greater than $\frac{1}{2}$, or if there is a jump strictly greater than 1 between two consecutive coordinates. Because $O(k+1, k)$ and $\mathrm{SO}(k+1, k)_{0}$ have the same spherical complementary series, Corollary 3 implies that an analogous result must be true for $\mathrm{SO}(n+1, n)$.

Proposition 10. Let $G=\mathrm{SO}(n+1, n)$ and let $v=\left(v_{1}, \ldots, v_{n}\right)$ be a real character of $A$. We may assume that

$$
v_{1} \geq \cdots \geq v_{p} \geq 0 \quad \text { and } \quad v_{p+1} \geq \cdots \geq v_{n} \geq 0 .
$$

Suppose that any of the following conditions holds:

(1) $v_{p}>\frac{1}{2}$ or $v_{n}>\frac{1}{2}$, or

(2) $v_{i}-v_{i+1}>1$, for some $i$ with $1 \leq i \leq p-1$, or $p+1 \leq i \leq n-1$.

Then the Langlands quotient $J\left(\delta^{p, q}, v\right)$ of $\mathrm{SO}(n+1, n)$ is not unitary.

\section{A matching of petite $K$-types with relevant $W^{\delta}$-types}

Given $\delta^{p, q}$ as in the previous section, recall the stabilizer of $\delta^{p, q}$ in $W$

$$
W^{\delta^{p, q}} \simeq W\left(B_{p}\right) \times W\left(B_{q}\right) \subseteq W\left(B_{n}\right) .
$$

We let $W\left(B_{p}\right)$ act on the first $p$ coordinates, and $W\left(B_{q}\right)$ on the last $q$ coordinates.

The $M$-type $\delta^{p, q}$ is contained in the (unique) fine $K$-type

$$
\mu_{\delta^{p, q}}=\operatorname{triv} \otimes \Lambda^{q}\left(\mathbb{C}^{n}\right) .
$$

Here $\mathbb{C}^{n}$ represents the standard representation of $O(n)$ with (standard) basis $\left\{v_{1}, v_{2}, \ldots, v_{n}\right\}$. For every $K$-type $\mu$ whose restriction to $M$ contains $\delta^{p, q}$, we denote by $E_{\mu}$ the vector space carrying the $K$-type $\mu$ and by $E_{\mu}\left(\delta^{p, q}\right)$ the isotypic component of the $M$-type $\delta^{p, q}$ inside $E_{\mu}$. In particular, when $\mu$ is the fine $K$-type $\mu_{\delta^{p, q}, \text { we set }}$

$$
E_{\mu_{\delta}, q}=\Lambda^{q}\left(\mathbb{C}^{n}\right),
$$

and we let $E_{\mu_{\delta} p, q}\left(\delta^{p, q}\right)$ be the one-dimensional space of $\Lambda^{q}\left(\mathbb{C}^{n}\right)$ spanned by the vector

$$
u=v_{p+1} \wedge v_{p+2} \wedge \cdots \wedge v_{n}
$$


Given the $M$-type $\delta^{p, q}$ and a $K$-type $\mu$ containing $\delta^{p, q}$, one can look at the representation $\psi_{\mu}$ of $W^{\delta^{p, q}}$ on $\operatorname{Hom}_{M}\left(\mu, \delta^{p, q}\right)$, or, equivalently, on

$$
V_{\mu}\left[\delta^{p, q}\right]:=\operatorname{Hom}_{\mathbb{C}}\left(E_{\mu}\left(\delta^{p, q}\right), E_{\mu_{\delta}^{p, q}}\left(\delta^{p, q}\right)\right) .
$$

For each $[\sigma] \in W^{\delta^{p, q}}$ we choose a representative $\sigma$ in $K$. Then, for $T \in V_{\mu}\left[\delta^{p, q}\right]$, we define $\psi_{\mu}([\sigma])(T)$ to be the map from $E_{\mu}\left(\delta^{p, q}\right)$ to $E_{\mu_{\delta} p, q}\left(\delta^{p, q}\right)$ given by

$$
\left(\psi_{\mu}([\sigma])(T)\right)(w)=\mu_{\delta^{p, q}}(\sigma)\left(T\left(\mu\left(\sigma^{-1}\right) w\right)\right) \text { for all } w \in E_{\mu}\left(\delta^{p, q}\right) .
$$

We are interested in computing the set of representations $\psi_{\mu}$ associated to petite $K$-types $\mu$.

The notion of "petite" $K$-type for real split groups is carefully explained in [Barbasch et al. 2008, Sections 4.5 and 4.6]. For the purpose of this paper, it is sufficient to consider $K$-types of level at most 2, which are necessarily petite. We recall the definition of the "level" of a $K$-type. Recall the elements $Z_{\alpha}$ from Section 2.

Definition 11 [Adams et al. 2007, Section 4]. An irreducible representation $\mu$ of $K$ is said to be level $k$ if $|\gamma| \leq k$ for every root $\alpha$ and every eigenvalue $\gamma$ of $d \mu\left(i Z_{\alpha}\right)$.

Recall that $W^{\delta^{p, q}}$ is isomorphic to the Weyl group of the group

$$
G^{\delta^{p, q}}=\mathrm{SO}(p+1, p)_{0} \times \mathrm{SO}(q+1, q)_{0} .
$$

The relevant $W^{\delta^{p, q}}$-types are a minimal set of irreducible representations of $W^{\delta^{p, q}}$ that detect nonunitarity for spherical Langlands quotients of $G^{\delta^{p, q}}$ (see (3-2)).

Theorem 12 [Barbasch 2004]. The following is a set of relevant $W\left(B_{k}\right)$-types for the group $\mathrm{SO}(k+1, k)_{0}$ :

$$
\{(k-m, m) \times(0): 0 \leq m \leq[k / 2]\} \cup\{(k-m) \times(m): 0 \leq m \leq k\} .
$$

Relevant $W\left(B_{p}\right) \times W\left(B_{q}\right)$-types of $\mathrm{SO}(p+1, p)_{0} \times \mathrm{SO}(q+1, q)_{0}$ are of the form

$\psi \otimes$ triv $\quad$ or $\quad \operatorname{triv} \otimes \tau$

with $\psi$ and $\tau$ a relevant $W$-type for $\mathrm{SO}(p+1, p)_{0}$ and $\mathrm{SO}(q+1, q)_{0}$, respectively.

The parametrization of $W\left(B_{k}\right)$-types in terms of pairs of partitions can be found, for example, in [Pantano et al. 2010, Section 9]. We give here just a short description of the $W\left(B_{k}\right)$-types we need. Recall that $W\left(B_{k}\right)$ is a semidirect product of the symmetric group $S_{k}$ by the abelian normal subgroup $(\mathbb{Z} / 2 \mathbb{Z})^{k}$. The irreducible representations of $S_{k}$ are parametrized by partitions of $k$, with the trivial partition $(k)$ corresponding to the trivial representation of $S_{k}$. If $c+d=k$, with $d \leq c$, then the symbol $(c, d) \times(0)$ (or simply $(c, d)$ ) denotes the pullback to $W\left(B_{k}\right)$ of the 
irreducible representation of $S_{k}$ corresponding to the partition $(c, d)$. This representation is a summand of dimension $\left(\begin{array}{l}k \\ c\end{array}\right)(k-2 c+1) /(k-c+1)$ of the permutation module $M^{(c, d)}=\operatorname{Ind}_{S_{c} \times S_{d}}^{S_{k}}$ (triv), with $(\mathbb{Z} / 2 \mathbb{Z})^{k}$ acting trivially.

If $a$ and $b$ are nonnegative integers with $a+b=k$ then the symbol $(a) \times(b)$ denotes the irreducible representation of $W\left(B_{k}\right)$ of dimension $\left(\begin{array}{l}k \\ a\end{array}\right)$ induced from the one-dimensional representation of $\left(S_{a} \times S_{b}\right) \ltimes(\mathbb{Z} / 2 \mathbb{Z})^{k}$ in which $S_{a}, S_{b}$, and $(\mathbb{Z} / 2 \mathbb{Z})^{a}$ act trivially, and $(\mathbb{Z} / 2 \mathbb{Z})^{b}$ acts by sign. Here $\left(S_{a} \times S_{b}\right)$ is the stabilizer in $S_{k}$ of the character (triv) $)^{a} \otimes(\operatorname{sign})^{b}$ of $(\mathbb{Z} / 2 \mathbb{Z})^{k}$.

Theorem 9 asserts that, for every $M$-type $\delta^{p, q}$ of $\mathrm{SO}(n+1, n)$ and every relevant $W^{\delta^{p, q}}$-type $\psi$, there exists a $K$-type $\mu$ of level at most 2 such that

$$
\psi_{\mu} \cong \psi
$$

We now describe the matching explicitly. We may restrict our attention to the case $p \geq q$. Indeed, for all choices of the parameters, we have

$$
J\left(\delta^{q, p},\left(v^{q} \mid v^{p}\right)\right) \simeq J\left(\delta^{p, q},\left(v^{p} \mid v^{q}\right)\right) \otimes \chi,
$$

with $\chi$ the nontrivial unitary character of $\mathrm{SO}(n+1, n)$.

The matching is presented in Table 1 (and will be proved in the next few sections).

Here we use partitions to parametrize some of the representations of $O(n)$ (and later of $O(n, \mathbb{C})$ and $\operatorname{GL}(n, \mathbb{C})$ ). See, for example, [Fulton and Harris 1991, Lectures 6 and 19]. For example, if the partition $\lambda=\left(\lambda_{1}, \ldots \lambda_{k}\right)$ has at most $n / 2$ parts, it parametrizes a representation of $O(n)$ with highest weight $\left(\lambda_{1}, \ldots, \lambda_{k}, 0, \ldots, 0\right)$.

Remark 13. All $K$-types recorded in this table are level at most 2.

Proof. By definition, fine $K$-types have level at most 1 ; hence every irreducible constituent of the tensor product of two fine $K$-types is of level at most two. This

$$
\begin{array}{cc}
\text { The relevant } W^{\delta^{p, q}} \text {-type } \psi & \text { A petite } K \text {-type } \mu \text { such that } \psi_{\mu}=\psi \\
((p-k) \times(k)) \otimes \text { triv } & \Lambda^{k}\left(\mathbb{C}^{n+1}\right) \otimes \Lambda^{q+k}\left(\mathbb{C}^{n}\right) \\
(p-k, k) \otimes \operatorname{triv} & \operatorname{Res}_{S(O(n+1) \times O(n))}^{O(n+1, \mathbb{C}) \times O(n, \mathbb{C})}\left[\operatorname{triv} \otimes\left(V_{\left(2^{k}, 1^{q}\right)}^{O(n)}\right)\right] \\
\operatorname{triv} \otimes((q-k) \times(k)) & \Lambda^{k}\left(\mathbb{C}^{n+1}\right) \otimes \Lambda^{q-k}\left(\mathbb{C}^{n}\right) \\
\operatorname{triv} \otimes(q-k, k) & \operatorname{Res}_{S(O(n+1) \times O(n))}^{O(n+1, \mathbb{C}) \times O(n, \mathbb{C})}\left[\operatorname{triv} \otimes\left(V_{\left(2^{k}, 1^{q-2 k}\right)}^{O(n)}\right)\right]
\end{array}
$$

Table 1. Matching. 
implies, for example, that the $K$-types

$\operatorname{Res}_{S(O(n+1) \times O(n))}^{O(n+1, \mathbb{C}) \times O(n, \mathbb{C})}\left[\operatorname{triv} \otimes\left(V_{\left(2^{k}, 1^{q-2 k}\right)}^{O(n)}\right)\right] \subseteq\left[\operatorname{triv} \otimes \Lambda^{k}\left(\mathbb{C}^{n}\right)\right] \otimes\left[\operatorname{triv} \otimes \Lambda^{q-k}\left(\mathbb{C}^{n}\right)\right]$ and

$\operatorname{Res}_{S(O(n+1) \times O(n))}^{O(n+1, \mathbb{C}) \times O(n, \mathbb{C})}\left[\operatorname{triv} \otimes\left(V_{\left(2^{k}, 1^{q}\right)}^{O(n)}\right)\right] \subseteq\left[\operatorname{triv} \otimes \Lambda^{k}\left(\mathbb{C}^{n}\right)\right] \otimes\left[\operatorname{triv} \otimes \Lambda^{q+k}\left(\mathbb{C}^{n}\right)\right]$

are level at most 2. Every $K$-type of the form

$$
\Lambda^{a}\left(\mathbb{C}^{n+1}\right) \otimes \Lambda^{b}\left(\mathbb{C}^{n}\right)
$$

is also level at most 2 , since $Z_{\epsilon_{k}}$ acts trivially on $\Lambda^{b}\left(\mathbb{C}^{n}\right)$ and it acts on $\Lambda^{a}\left(\mathbb{C}^{n+1}\right)$ with eigenvalues $0, \pm 2 i$ and $Z_{\epsilon_{i} \pm \epsilon_{j}}$ acts on both $\Lambda^{b}\left(\mathbb{C}^{n}\right)$ and $\Lambda^{a}\left(\mathbb{C}^{n+1}\right)$ with eigenvalues $0, \pm i$.

\subsection{The $W\left(B_{q}\right)$-type $(q-k) \times(k)$. Consider the $K$-type}

$$
\mu:=\Lambda^{k}\left(\mathbb{C}^{n+1}\right) \otimes \Lambda^{q-k}\left(\mathbb{C}^{n}\right),
$$

where $\mathbb{C}^{n+1}$ and $\mathbb{C}^{n}$ are the standard representations of $O(n+1)$ and $O(n)$, respectively, with bases $\left\{e_{1}, e_{2}, \ldots, e_{n+1}\right\}$ and $\left\{v_{1}, v_{2}, \ldots, v_{n}\right\}$.

The restriction of $\mu$ to $M$ contains the $M$-type $\delta^{p, q}$ with multiplicity $\left(\begin{array}{l}q \\ k\end{array}\right)$. The $\delta^{p, q}$-isotypic component inside $\mu$ is spanned by the vectors:

$$
w_{J}:=e_{q+2-i_{k}} \wedge e_{q+2-i_{k-1}} \wedge \cdots \wedge e_{q+2-i_{1}} \otimes v_{p+j_{1}} \wedge v_{p+j_{2}} \wedge \cdots \wedge v_{p+j_{q-k}},
$$

where $J=\left\{1 \leq j_{1}<j_{2}<\cdots<j_{q-k} \leq q\right\}$ is a subset of $\{1,2, \ldots, q\}$ of cardinality $q-k$, and $I=\left\{1 \leq i_{1}<i_{2}<\cdots<i_{k} \leq q\right\}$ is the complement of $J$ in the same set. Indeed one can check that

$$
T\left(t_{1}, t_{2}, \ldots, t_{n}\right) \cdot w_{J}=\delta^{p, q}\left(T\left(t_{1}, t_{2}, \ldots, t_{n}\right)\right) \cdot w_{J}
$$

for all $T\left(t_{1}, t_{2}, \ldots, t_{n}\right)$ in $M$, and that this is the entire $\delta^{p, q}$-isotypic subspace of $\mu$.

We study the representation $\psi_{\mu}$ of $W^{\delta^{p, q}}$ on the $\left(\begin{array}{l}q \\ k\end{array}\right)$-dimensional space

$$
V_{\mu}\left[\delta^{p, q}\right]=\operatorname{Hom}_{M}\left(\mu, \delta^{p, q}\right) .
$$

For each $L=\left\{l_{1}<l_{2}<\cdots<l_{q-k}\right\} \subset\{1,2, \ldots, q\}$, set

$$
T_{L}\left(w_{J}\right)= \begin{cases}u & \text { if } J=L \\ 0 & \text { otherwise }\end{cases}
$$

Recall that the vector $u=v_{p+1} \wedge v_{p+2} \wedge \cdots \wedge v_{n}$ is a basis for the $\delta^{p, q}$-isotypic inside $\mu_{\delta^{p, q}}$. The maps $\left\{T_{L}\right\}$ form a basis of $V_{\mu}\left[\delta^{p, q}\right]$. Note that

$$
\sigma_{\epsilon_{l}} \cdot v_{s}=+v_{s} \text { and } \sigma_{\epsilon_{l}} \cdot e_{t}= \begin{cases}-e_{t} & \text { if } t=1 \text { or } t=n+2-l, \\ +e_{t} & \text { otherwise, }\end{cases}
$$


for all $l, s=1, \ldots, n$ and $t=1, \ldots, n+1$. Hence:

- $\mu_{\delta, q}\left(\sigma_{\epsilon_{l}}\right) u=u$.

- $\mu\left(\sigma_{\epsilon_{l}}\right) w_{J}=\mu\left(\sigma_{\epsilon_{l}}^{-1}\right) w_{J}= \begin{cases}-w_{J} & \text { if } l \in p+\{1,2, \ldots, q\} \backslash J, \\ +w_{J} & \text { otherwise, }\end{cases}$

and consequently

$$
\psi_{\mu}\left(s_{\epsilon_{l}}\right) T_{J}= \begin{cases}-T_{J} & \text { if } l \in p+\{1,2, \ldots, q\} \backslash J, \\ +T_{J} & \text { otherwise. }\end{cases}
$$

Similarly we observe that

$$
\sigma_{\epsilon_{l}-\epsilon_{l+1}} \cdot v_{s}= \begin{cases}v_{s+1} & \text { if } s=l \\ -v_{s-1} & \text { if } s=l+1 \\ v_{s} & \text { if } s \neq l, l+1\end{cases}
$$

and

$$
\sigma_{\epsilon_{l}-\epsilon_{l+1}} \cdot e_{t}= \begin{cases}+e_{t-1} & \text { if } t=n+2-l, \\ -e_{t+1} & \text { if } t=n+2-(l+1), \\ +e_{t} & \text { if } t \neq n+2-l, n+2-(l+1)\end{cases}
$$

for $s=1, \ldots, n, t=1, \ldots, n+1$ and $l=1, \ldots, p-1$ or $l=p+1, \ldots, n-1$. Hence:

- $\mu_{\delta^{p, q}}\left(\sigma_{\epsilon_{l}-\epsilon_{l+1}}\right) u=u$.

$$
\text { - } \mu\left(\sigma_{\epsilon_{l}-\epsilon_{l+1}}\right) w_{J}= \begin{cases}+w_{J} & \text { if } l<p, \\ +w_{J} & \text { if } l>p \text { and either } l, l+1 \in p+J \\ & \text { or } l, l+1 \in p+\{1,2, \ldots, q\} \backslash J, \\ -w_{J \triangle\{l-p, l+1-p\}} & \text { otherwise, }\end{cases}
$$

and since $\mu\left(\sigma_{\epsilon_{l}-\epsilon_{l+1}}\right) w_{J}=\mu\left(\sigma_{\epsilon_{l}-\epsilon_{l+1}}^{-1}\right) w_{J}$,

(4-14) $\psi_{\mu}\left(\sigma_{\epsilon_{l}-\epsilon_{l+1}}\right) T_{J}= \begin{cases}+T_{J} & \text { if } l<p, \\ +T_{J} & \text { if } l>p \text { and either } l, l+1 \in p+J \\ & \text { or } l, l+1 \in p+\{1,2, \ldots, q\} \backslash J, \\ -T_{J \triangle\{l-p, l+1-p\}} & \text { otherwise. }\end{cases}$

This information is enough to characterize the representation $\psi_{\mu}$ of $W^{\delta^{p, q}}$ on $V_{\mu}\left[\delta^{p, q}\right]$. Recall that $W\left(B_{p}\right)$ and $W\left(B_{q}\right)$ are realized as the (appropriate) subgroups of $W\left(B_{n}\right)$ acting on the coordinates $\{1, \ldots, p\}$ and $\{p+1, \ldots, n\}$, respectively. Let $S_{q-k}$ and $(\mathbb{Z} / 2 \mathbb{Z})^{q-k}$ be the subgroups of $W\left(B_{q}\right)$ acting on the first $q-k$ coordinates $\{p+1, p+2, \ldots, n-k\}$, and let $S_{k},(\mathbb{Z} / 2 \mathbb{Z})^{k}$ be the ones acting on the last $k$ coordinates $\{n-k+1, n-k+2, \ldots, n\}$. Here the $\mathbb{Z} / 2 \mathbb{Z}$ factors are generated by the $\sigma_{\epsilon_{l}}$, and the symmetric groups by the $\sigma_{\epsilon_{l}-\epsilon_{l+1}}$. Note that: 
- The restriction of $\psi_{\mu}$ to $W\left(B_{p}\right)$ is trivial, hence $\psi_{\mu}$ is of the form triv $\otimes \psi^{\prime}$ for some representation $\psi^{\prime}$ of $W\left(B_{q}\right)$.

- The groups $S_{q-k}, S_{k}$ and $(\mathbb{Z} / 2 \mathbb{Z})^{q-k}$ act trivially on the vector $T_{\{1, \ldots, q-k\}}$, and $(\mathbb{Z} / 2 \mathbb{Z})^{k}$ acts on it by sign. Hence the restriction of $\psi^{\prime}$ to the group $\left(S_{q-k} \times S_{k}\right) \ltimes(\mathbb{Z} / 2 \mathbb{Z})^{q}$ contains the one-dimensional representation

$$
[(q-k) \otimes(k)] \cdot\left[(\text { triv })^{q-k} \otimes(\operatorname{sign})^{k}\right] .
$$

By Frobenius reciprocity, $\psi^{\prime}$ contains the irreducible representation

$$
(q-k) \times(k):=\operatorname{Ind}_{\left(S_{q-k} \times S_{k}\right) \ltimes(\mathbb{Z} / 2 \mathbb{Z})^{q}}^{W\left(B_{q}\right)}[(q-k) \otimes(k)] \cdot\left[(\text { triv })^{q-k} \otimes(\text { sign })^{k}\right] .
$$

Actually $\psi^{\prime}=(q-k) \times(k)$ for dimensional reasons.

We conclude that $\psi_{\mu}=\operatorname{triv} \otimes((q-k) \times(k))$, as claimed.

4.2. The $\boldsymbol{W}\left(\boldsymbol{B}_{q}\right)$-type $(\boldsymbol{q}-\boldsymbol{k}, \boldsymbol{k})$. Consider the (possibly reducible) representation

$$
\mu:=\operatorname{triv} \otimes\left[\Lambda^{k}\left(\mathbb{C}^{n}\right) \otimes \Lambda^{q-k}\left(\mathbb{C}^{n}\right)\right]
$$

of $K$, where triv denotes the trivial representation of $O(n+1)$ and $\mathbb{C}^{n}$ denotes the standard representation of $O(n)$ (with basis $\left\{v_{1}, v_{2}, \ldots, v_{n}\right\}$ ).

Note that $\mu$ contains the $M$-type $\delta^{p, q}$ with multiplicity $\left(\begin{array}{l}q \\ k\end{array}\right)$. For every subset $J=\left\{1 \leq j_{1}<j_{2}<\cdots<j_{q-k} \leq q\right\}$, let $I=\left\{i_{1}<i_{2}<\cdots<i_{k}\right\}$ be its complement in the set $\{1,2, \ldots, q\}$, and let

$$
w_{J}:=v_{p+i_{1}} \wedge v_{p+i_{2}} \wedge \cdots \wedge v_{p+i_{k}} \otimes v_{p+j_{1}} \wedge v_{p+j_{2}} \wedge \cdots \wedge v_{p+j_{q-k}} .
$$

Then the vectors $\left\{w_{J}\right\}$ span the $\delta^{p, q}$-isotypic component inside $\mu$.

We study the representation $\psi_{\mu}$ of $W^{\delta^{p, q}}$ on the $\left(\begin{array}{l}q \\ k\end{array}\right)$-dimensional space $V_{\mu}\left[\delta^{p, q}\right]$. For all $L=\left\{1 \leq l_{1}<l_{2}<\cdots<l_{q-k} \leq q\right\}$, set

$$
T_{L}\left(w_{J}\right)= \begin{cases}u & \text { if } J=L, \\ 0 & \text { otherwise }\end{cases}
$$

Then the maps $\left\{T_{L}\right\}$ form a basis of $V_{\mu}\left[\delta^{p, q}\right]$. Note that

$$
\sigma_{\epsilon_{l}} \cdot v_{s}=+v_{s} \text { for all } l, s=1, \ldots, n ;
$$

hence

$$
\mu_{\delta^{p, q}}\left(\sigma_{\epsilon_{l}}\right) u=u \quad \text { and } \quad \mu\left(\sigma_{\epsilon_{l}}\right) w_{J}=w_{J} \quad \text { for all } J .
$$

This implies that

$$
\psi_{\mu}\left(s_{\epsilon_{l}}\right) T_{J}=T_{J} \text { for all } J
$$

so $\psi_{\mu}$ is really a representation of $S_{p} \times S_{q}$. Next we show that $S_{p}$ acts trivially. 
Recall from (4-12) that for all $s=1, \ldots, n$ and all $l=1, \ldots, p-1$ or $l=p+1$, $\ldots, n-1$, we have

$$
\sigma_{\epsilon_{l}-\epsilon_{l+1}} \cdot v_{s}= \begin{cases}v_{s+1} & \text { if } s=l \\ -v_{s-1} & \text { if } s=l+1 \\ v_{s} & \text { if } s \neq l, l+1\end{cases}
$$

Hence:

- $\mu_{\delta^{p}, q}\left(\sigma_{\epsilon_{l}-\epsilon_{l+1}}\right) u=u$.

- $\mu\left(\sigma_{\epsilon_{l}-\epsilon_{l+1}}\right) w_{J}= \begin{cases}+w_{J} & \text { if } l<p, \\ +w_{J} & \text { if } l>p \text { and either } l, l+1 \in p+J \\ & \text { or } l, l+1 \in p+\{1,2, \ldots, q\} \backslash J, \\ -w_{J \triangle\{l-p, l+1-p\}} & \text { otherwise, }\end{cases}$

and since $\mu\left(\sigma_{\epsilon_{l}-\epsilon_{l+1}}\right) w_{J}=\mu\left(\sigma_{\epsilon_{l}-\epsilon_{l+1}}^{-1}\right) w_{J}$,

$$
\psi_{\mu}\left(\sigma_{\epsilon_{l}-\epsilon_{l+1}}\right) T_{J}= \begin{cases}+T_{J} & \text { if } l<p, \\ +T_{J} & \text { if } l>p \text { and either } l, l+1 \in p+J \\ & \text { or } l, l+1 \in p+\{1,2, \ldots, q\} \backslash J, \\ -T_{J \triangle\{l-p, l+1-p\}} & \text { otherwise. }\end{cases}
$$

Therefore $S_{p}$ acts indeed trivially, so $\psi_{\mu}$ is of the form triv $\otimes \psi^{\prime}$ for some representation $\psi^{\prime}$ of $S_{q}$. Finally we prove that $\psi^{\prime}$ equals the permutation module $\operatorname{Ind}_{S_{q-k} \times S_{k}}^{S_{q}} \operatorname{triv}=M^{(q-k, k)}$. Write

$$
\operatorname{Hom}_{M}\left(\mu, \delta^{p, q}\right)=\bigoplus_{\substack{L \subseteq\{1, \ldots, q\} \\|L|=q-k}} U_{L},
$$

with $U_{L}:=\mathbb{C} T_{L}$. The symmetric group $S_{q}$ permutes the subspaces $U_{L}$ transitively. Set $L_{0}:=\{1,2, \ldots, q-k\}$ and $U_{0}:=U_{L_{0}}$, and let $H$ be the stabilizer of $U_{0}$ in $S_{q}$ (i.e., the set of all $\eta$ in $S_{q}$ such that $\eta U_{0}=U_{0}$ ). Note that

- $H \simeq S_{q-k} \times S_{k}$. (We identify $S_{q-k}$ and $S_{k}$ with the subgroups of $S_{q}$ acting on the first $q-k$ coordinates and the last $k$ coordinates, respectively.)

- $U_{0}$ is stable under $H$ and carries the trivial representation of $H$.

- The $S_{q}$-module $V_{\mu}\left[\delta^{p, q}\right]$ is induced from the $H$-module $U_{0}$ (see [Serre 1977, Proposition 19]).

Therefore

$$
\psi^{\prime}=\operatorname{Ind}_{S_{q-k} \times S_{k}}^{S_{q}} \operatorname{triv}=M^{(q-k, k)},
$$

and $\psi_{\mu}=\operatorname{triv} \otimes M^{(q-k, k)}$. 
The representation triv $\otimes M^{(q-k, k)}$ is clearly reducible because the permutation module $M^{(q-k, k)}$ of $S_{q}$ decomposes as a direct sum of Specht modules

$$
M^{(q-k, k)}=\bigoplus_{\lambda \unrhd(q-k, k)} \mathscr{\varphi}^{\lambda}=\bigoplus_{a=0}^{k} \mathscr{S}^{(q-a, a)} .
$$

Here $\unrhd$ denotes the dominance (partial) ordering on partitions: If $\lambda=\left(\lambda_{1}, \lambda_{2}, \ldots\right)$ and $\mu=\left(\mu_{1}, \mu_{2}, \ldots\right)$ are partitions of $N$ then $\lambda \unrhd \mu$ if $\sum_{i=1}^{r} \lambda_{i} \geq \sum_{i=1}^{r} \mu_{i}$ for all $r$. With abuse of notation, we let $(q-0,0)$ denote the trivial partition. Then

$$
\operatorname{triv} \otimes M^{(q-k, k)}=\bigoplus_{a=0}^{k} \operatorname{triv} \otimes(q-a, a) .
$$

The module $\psi_{\mu}=V_{\mu}\left[\delta^{p, q}\right]$ is also reducible because the representation $\mu$ of $K$ decomposes as a direct sum of $K$-types. We need to identify the irreducible component of $\mu$ containing $(q-k, k)$.

The first task is to compute the decomposition of the tensor product

$$
\Lambda^{k}\left(\mathbb{C}^{n}\right) \otimes \Lambda^{q-k}\left(\mathbb{C}^{n}\right)
$$

into $O(n)$-types. We do this in three steps: First we decompose $\Lambda^{k}\left(\mathbb{C}^{n}\right) \otimes \Lambda^{q-k}\left(\mathbb{C}^{n}\right)$ into irreducible representations of $\operatorname{GL}(n, \mathbb{C})$. Then we decompose each $\operatorname{GL}(n, \mathbb{C})$ type occurring in such a decomposition as a direct sum of $O(n, \mathbb{C})$-types. Finally we restrict to $O(n)$.

We will use Weyl's construction of irreducible representations of $\operatorname{GL}(n, \mathbb{C})$ and $O(n, \mathbb{C})$ (see, for example, [Fulton and Harris 1991, Lectures 6 and 19]). By Pieri's formula,

$$
\Lambda^{k}\left(\mathbb{C}^{n}\right) \otimes \Lambda^{q-k}\left(\mathbb{C}^{n}\right)=V_{\left(1^{k}\right)}^{\mathrm{GL}(n)} \otimes V_{\left(1^{q-k}\right)}^{\mathrm{GL}(n)}=\bigoplus_{a=0}^{k} V_{\left(2^{a}, 1^{q-2 a}\right)}^{\mathrm{GL}(n)}
$$

Note that the partitions $\left(2^{a}, 1^{q-2 a}\right)$ have at most

$$
a+(q-2 a)=q-a \leq q \leq n / 2
$$

parts, so we can apply Littlewood's restriction formula [1944]:

$$
\begin{gathered}
\operatorname{Res}_{O(n, \mathbb{C})}^{\mathrm{GL}(n, \mathbb{C})} V_{\left(2^{a}, 1^{q-2 a}\right)}^{\mathrm{GL}(n)} \\
=\bigoplus_{v: \text { \#parts } \leq n / 2}\left(\sum_{\xi: \text { even parts }} N_{v, \xi,\left(2^{a}, 1^{q-2 a}\right)}\right) V_{v}^{O(n)} \\
=\bigoplus_{b=0}^{a} \underbrace{N_{\left(2^{b}, 1^{q-2 a}\right),\left(2^{a-b}\right),\left(2^{a}, 1^{q-2 a}\right)}}_{=1} V_{\left(2^{b}, 1^{q-2 a}\right)}^{O(n)}=\bigoplus_{b=0}^{a} V_{\left(2^{b}, 1^{q-2 a}\right)}^{O(n)} .
\end{gathered}
$$


Here the $N_{\nu, \xi, \lambda}$ are the Littlewood-Richardson numbers. We deduce that

$$
\Lambda^{k}\left(\mathbb{C}^{n}\right) \otimes \Lambda^{q-k}\left(\mathbb{C}^{n}\right)=\bigoplus_{a=0}^{k}\left(\bigoplus_{b=0}^{a} V_{\left(2^{b}, 1^{q-2 a}\right)}^{O(n)}\right)
$$

(as a representation of $O(n, \mathbb{C})$ ), and

$$
\begin{aligned}
\mu & :=\operatorname{triv} \otimes\left[\Lambda^{k}\left(\mathbb{C}^{n}\right) \otimes \Lambda^{q-k}\left(\mathbb{C}^{n}\right)\right] \\
& =\bigoplus_{a=0}^{k}\left(\bigoplus_{b=0}^{a} \operatorname{Res}_{S(O(n+1) \times O(n))}^{O(n+1, \mathbb{C}) \times O(n, \mathbb{C})} \operatorname{triv} \otimes V_{\left(2^{b}, 1^{q-2 a}\right)}^{O(n)}\right)
\end{aligned}
$$

(as a representation of $K=S(O(n+1) \times O(n)))$. Note that the $K$-representation

$$
\operatorname{Res}_{S(O(n+1) \times O(n))}^{O(n+1, \mathbb{C}) \times O(n, \mathbb{C})} \operatorname{triv} \otimes V_{\left(2^{b}, 1^{q-2 a}\right)}^{O(n)}
$$

embeds in the tensor product

$$
\left[\operatorname{triv} \otimes \Lambda^{b}\left(\mathbb{C}^{n}\right)\right] \otimes\left[\operatorname{triv} \otimes \Lambda^{q-2 a+b}\left(\mathbb{C}^{n}\right)\right] .
$$

Every $M$-type $\delta^{r, s}$ appearing in this tensor product satisfies

$$
s \leq q-2 a+2 b \leq q
$$

hence $\delta^{r, s} \neq \delta^{p, q}$ for all $b \neq a$, and

(4-37) $\operatorname{Hom}_{M}\left(\operatorname{Res}_{S(O(n+1) \times O(n))}^{O(n+1, \mathbb{C}) \times O(n, \mathbb{C})} \operatorname{triv} \otimes V_{\left(2^{b}, 1^{q-2 a}\right)}^{O(n)}, \delta^{p, q}\right)=\{0\} \quad$ for all $b \neq a$.

It follows that, as a representation of $W^{\delta^{p, q}}$,

$$
\begin{aligned}
\psi_{\mu} & =\operatorname{Hom}_{M}\left(\mu, \delta^{p, q}\right) \\
& =\bigoplus_{a=0}^{k}\left(\bigoplus_{b=0}^{a} \operatorname{Hom}_{M}\left(\operatorname{Res}_{S(O(n+1) \times O(n))}^{O(n+1, \mathbb{C}) \times O(n, \mathbb{C})} \operatorname{triv} \otimes V_{\left(2^{b}, 1^{-2 a}\right)}^{O(n)}, \delta^{p, q}\right)\right) \\
& =\bigoplus_{a=0}^{k} \operatorname{Hom}_{M}\left(\operatorname{Res}_{S(O(n+1) \times O(n))}^{O(n+1, \mathbb{C}) \times O(n, \mathbb{C})} \operatorname{triv} \otimes V_{\left(2^{a}, 1^{q-2 a}\right)}^{O(n)}, \delta^{p, q}\right) .
\end{aligned}
$$

We also know that

$$
\psi_{\mu}=\bigoplus_{a=0}^{k} \operatorname{triv} \otimes(q-a, a) .
$$

Equations (4-38) and (4-39) hold for all $k=0, \ldots,[q / 2]$. A simple induction argument shows that

(4-40) $\quad \operatorname{Hom}_{M}\left(\operatorname{Res}_{S(O(n+1) \times O(n))}^{O(n+1, \mathbb{C}) \times O(n, \mathbb{C})} \operatorname{triv} \otimes V_{\left(2^{a}, 1^{q-2 a}\right)}^{O(n)}, \delta^{p, q}\right)=\operatorname{triv} \otimes(q-a, a)$, 
for all $a=1 \ldots k$. In particular,

$$
\operatorname{Hom}_{M}\left(\operatorname{Res}_{S(O(n+1) \times O(n))}^{O(n+1, \mathbb{C}) \times O(n, \mathbb{C})} \operatorname{triv} \otimes V_{\left(2^{k}, 1^{q-2 k}\right)}^{O(n)}, \delta^{p, q}\right)=\operatorname{triv} \otimes(q-k, k) .
$$

4.3. The $W\left(B_{p}\right)$-types $(p-k, k)$ and $(p-k) \times(k)$. The calculations are similar to the ones done in Sections 4.2 and 4.1, respectively. We leave the details to the diligent reader.

\section{Some small rank examples}

In [Pantano et al. 2010], we proved Theorem 5 for metaplectic groups, except for the case $(p, q)=(2,2)$ and $v^{p}=v^{q}=\left(\frac{3}{2}, \frac{1}{2}\right)$. In this case, $J_{\mathrm{Mp}(8)}\left(\delta^{2,2}, v\right)$ coincides with the lowest $K$-type constituent of a weakly fair $A_{\mathfrak{q}}(\lambda)$ module with $\mathfrak{q}=\mathfrak{l}+\mathfrak{u}$, where $L \cong \widetilde{U}(2,2)$ and $\lambda=-\rho(\mathfrak{u})$. As a constituent of an $A_{\mathfrak{q}}(\lambda)$ module in the weakly fair range, it is unitary by a result of Vogan [1993] (see [Knapp and Vogan 1995, Theorem 0.54]). The unitarity of this representation will also follow from Theorem 6 since $v$ is a unipotent parameter (see Definition 27). This completes the proof of Theorem 5 for metaplectic groups.

In this section, we prove Theorem 5 for orthogonal groups as well. The proof is analogous to the one for the metaplectic groups.

For nonnegative integers $(p, q)$ such that $p+q \leq 4$, and parameters $v=\left(v^{p} \mid v^{q}\right)$ with $v^{p} \in \mathrm{CS}\left(\mathrm{SO}(p+1, p)_{0}, \delta_{0}\right)$ and $v^{q} \in \mathrm{CS}\left(\operatorname{SO}(q+1, q)_{0}, \delta_{0}\right)$, we need to show that $J\left(\delta^{p, q}, v\right)$ is unitary.

The main tool for the proof is unitary induction with deformation of parameters. In all cases except one, we think of $J\left(\delta^{p, q}, v\right)$ as an irreducible subquotient of an induced representation

$$
I\left(v^{q}\right)=\operatorname{Ind}_{P_{I}}^{G}\left(J\left(\delta^{p, 0}, v^{p}\right) \otimes \delta^{0, q} \otimes v^{q}\right),
$$

where $P_{I}=M_{I} A_{I} N_{I}$ is a parabolic subgroup of $G$ with Levi factor

$$
M_{I} A_{I} \cong \mathrm{SO}(p+1, p) \times \mathrm{GL}(1, \mathbb{R})^{q} .
$$

Here $\delta^{0, q}$ is the product of $q$ sign characters on the $\mathbb{Z} / 2 \mathbb{Z}$ parts of $\operatorname{GL}(1, \mathbb{R})$. The idea is to irreducibly deform the parameter $v^{q}$ to 0 . Since $J\left(\delta^{p, 0}, v^{p}\right)$ is unitary, and the Hermitian form on the induced representation can change signature only at reducibility points, this will prove that $J\left(\delta^{p, q}, v\right)$ is unitary. For this, we need to know when an induced representation stays irreducible under deformation.

Let $P=M A N$ be a minimal parabolic subgroup of $\mathrm{SO}(n+1, n)$, and let $P_{I}=$ $M_{I} A_{I} N_{I}$ be a parabolic subgroup containing $P$. Then

$$
P \cap M_{I}=M A_{M} N_{M}
$$


is a minimal parabolic subgroup of $M_{I}$, and $A=A_{M} A_{I}$. For each pair of (real) characters $v_{M} \in \mathfrak{a}_{M, \mathbb{R}}^{*}$ and $v_{I} \in \mathfrak{a}_{I, \mathbb{R}}^{*}$, write $v=\left(v_{M} \mid v_{I}\right)$ for the corresponding character of $A$. Let $\delta$ be a character of $M$, and let $J_{M_{I}}\left(\delta, v_{M}\right)$ be the Langlands subquotient of the principal series

$$
\operatorname{Ind}_{M A_{M} N_{M}}^{M_{I}}\left(\delta \otimes v_{M}\right) .
$$

Note that $J_{M_{I}}\left(\delta, v_{M}\right)$ is always irreducible.

Recall the notion of good and bad roots from page 484 (see (2-16)).

Proposition 14. Consider the induced representation

$$
I\left(v_{I}\right):=\operatorname{Ind}_{M_{I} A_{I} N_{I}}^{\mathrm{SO}(n+1, n)}\left(J_{M_{I}}\left(\delta, v_{M}\right) \otimes v_{I}\right)
$$

of $\operatorname{SO}(n+1, n)$. Set $v=\left(v_{M} \mid v_{I}\right)$, and assume that $v_{I}$ satisfies

$$
\begin{cases}\left\langle v, \beta^{\vee}\right\rangle \notin 2 \mathbb{Z}+1 & \text { for all } \beta \in \Delta\left(\mathfrak{n}_{I}\right) \text { that are good for } \delta, \\ \left\langle v, \beta^{\vee}\right\rangle \notin 2 \mathbb{Z}-\{0\} & \text { for all } \beta \in \Delta\left(\mathfrak{n}_{I}\right) \text { that are bad for } \delta .\end{cases}
$$

Then $I\left(v_{I}\right)$ is irreducible.

Proof. We claim that under the conditions (5-5), the operator $T\left(w_{0}, \mu, \delta^{p, q}, v\right)$ (see (3-3)) has no zero eigenvalues for any $K$-type $\mu$ containing $\delta^{p, q}$ and hence is invertible. This easily follows from [Barbasch et al. 2008, Theorem 2.10]. Then, as for the corresponding result for the metaplectic group [Pantano et al. 2010, Proposition 8.9], the proposition follows from (the proof of) [Knapp and Zuckerman 1977, Theorem 8] and [Speh and Vogan 1980, Corollary 3.9].

As for $\mathrm{Mp}(2 n)$ in [Pantano et al. 2010], we have the following consequence.

Corollary 15. In the setting of Proposition 14 , let $J\left(v_{I}\right)$ be the (irreducible) Langlands subquotient of $I\left(v_{I}\right)$. Let $R \subset \mathfrak{a}_{I, \mathbb{R}}^{*}$ be any connected region in the complement of the hyperplane arrangement defined in (5-5). If $J\left(v_{I}\right)$ is unitary for some value of $v_{I}$ in $R$, then $J(v)$ is unitary throughout the closure of $R$. In particular, for $v$ in the unit cube (i.e., if $0 \leq\left|v_{i}\right| \leq \frac{1}{2}$ for $\left.i=1 \ldots n\right), J(v)$ is unitary.

Theorem 16. Let $G=\mathrm{SO}(n+1, n)$. Choose $(p, q)$ with $p+q=n$, and let $v=$ $\left(v^{p} \mid v^{q}\right)$, with $v^{p}=\left(a_{1}, \ldots, a_{p}\right)$ and $v^{q}=\left(a_{p+1}, \ldots, a_{n}\right)$, be a character of $A$ such that

$$
v^{p} \in \mathrm{CS}\left(\operatorname{SO}(p+1, p)_{0}, \delta_{0}\right) \quad \text { and } \quad v^{q} \in \mathrm{CS}\left(\operatorname{SO}(q+1, q)_{0}, \delta_{0}\right) .
$$

\section{Suppose that}

- For all $j=1, \ldots$, , either $0 \leq\left|a_{j}\right| \leq 3 / 2$ or $a_{j} \in \mathbb{Z}+\frac{1}{2}$.

- For all $j=p+1, \ldots, n, 0 \leq\left|a_{j}\right| \leq \frac{1}{2}$ (i.e., $v^{q}$ belongs to the unit cube).

Then the Langlands subquotient $J\left(\delta^{p, q}, v\right)$ of $\mathrm{SO}(n+1, n)$ is unitary. 
Proof. The proof is completely analogous to the proof of [Pantano et al. 2010, Theorem 8.11]. If $P_{I}=M_{I} A_{I} N_{I}$ and $I\left(v^{q}\right)$ are as given in (5-1), then up to sign, the roots of $N_{I}$ are

$$
\begin{array}{ll}
\epsilon_{i} & \text { for } p+1 \leq i \leq n \\
\epsilon_{i} \pm \epsilon_{j} & \text { for } p+1 \leq i<j \leq n, \\
\epsilon_{i} \pm \epsilon_{j} & \text { for } 1 \leq i \leq p \text { and } p+1 \leq j \leq n .
\end{array}
$$

The roots in the first two rows are good for $\delta^{p, q}$, the remaining ones are bad. Under our assumptions on $v^{p}$, if $v^{q}$ is in the interior of the unit cube, then the conditions (5-5) are satisfied. By Proposition 14, $I\left(v^{q}\right)$ is irreducible. Moreover $J(0)=I(0)$ is unitary. By Corollary $15, J\left(\delta^{p, q}, v\right)$ is unitary for all $v^{q}$ in the (closed) unit cube.

The spherical complementary series parameters for $\mathrm{SO}(p+1, p)_{0}$ for $p=1,2,3$ are given at the end of Section 8. It is easily checked that they all satisfy the hypotheses of the theorem, and for $q=1,2$, the spherical complementary series parameters all belong to the unit cube, except for the isolated point $\left(\frac{3}{2}, \frac{1}{2}\right)$. Consequently Theorem 16 now implies the unitarity of all representations under consideration (for $n \leq 4)$ except $J\left(\delta^{2,2}, v\right)$, with $v^{p}=v^{q}=\left(\frac{3}{2}, \frac{1}{2}\right)$. Just as for the corresponding representation of $\operatorname{Mp}(8)$, we can realize it as a constituent of an $A_{\mathfrak{q}}(\lambda)$-module at the edge of the weakly fair range, which proves that it is unitary. See [Knapp and Vogan 1995, Example 3, Chapter VIII, §5] for a detailed discussion of this (reducible) module of $\mathrm{SO}(5,4)$. Note that its unitarity also follows from Theorem 29.

Collecting all these results, we have now proved Theorem 5.

\section{The theta correspondence}

For the case $p=n, q=0$, the authors of [Adams et al. 2007] use the theta correspondence to prove that if $v$ is a spherical unitary parameter for $\mathrm{SO}(n+1, n)$ then $v \in \operatorname{CS}\left(\operatorname{Mp}(2 n), \delta^{n, 0}\right)$. We will generalize the argument to relate the complementary series of the two families of groups to each other in more generality.

First we collect the facts about the theta correspondence that we need (some of them were already recalled in [Pantano et al. 2010]).

Let $\left(G, G^{\prime}\right)$ be a reductive dual pair in $\operatorname{Sp}(2 N, \mathbb{R})$, that is, $G$ and $G^{\prime}$ are reductive subgroups of $\operatorname{Sp}(2 N, \mathbb{R})$ which are mutual centralizers. Write $\widetilde{G}$ and $\widetilde{G}^{\prime}$ for the preimages of $G$ and $G^{\prime}$ in $\operatorname{Mp}(2 N)$ under the covering map. Howe [1989] defines a correspondence between irreducible representations of $\widetilde{G}$ and those of $\widetilde{G}^{\prime}$, and shows that this correspondence is a bijection between subsets of the genuine admissible duals of the two groups. Moreover subjugated to the correspondence is a bijection between $K$ - and $K^{\prime}$-types in the space of joint harmonics $\mathscr{H}$. Here $K$ and $K^{\prime}$ are maximal compact subgroups of $\widetilde{G}$ and $\widetilde{G}^{\prime}$, respectively. The $K$ - and $K^{\prime}$-types are 
assigned a degree and the correspondence satisfies the following property: If $\pi$ corresponds to $\pi^{\prime}$ in the correspondence for the dual pair $\left(G, G^{\prime}\right)$, and $\mu$ is a $K$ type of minimal degree occurring in $\pi$, then $\mu$ occurs in $\mathscr{H}$, and corresponds to a $K^{\prime}$-type $\mu^{\prime}$ which occurs in and is of minimal degree in $\pi^{\prime}$.

In general, the theta correspondence does not preserve unitarity. However we have preservation of unitarity in the stable range.

Definition 17. The dual pair $(O(r, s), \operatorname{Sp}(2 n, \mathbb{R}))$ is said to be in the stable range with $O(r, s)$ the smaller member if $n \geq r+s$. It is in the stable range with $\operatorname{Sp}(2 n, \mathbb{R})$ the smaller member if $\min \{r, s\} \geq 2 n$.

Theorem 18 [Li 1989c]. Suppose $\left(G, G^{\prime}\right)$ is a dual pair in the stable range with $G$ the smaller member. If $\pi$ is an irreducible genuine unitary representation of $\widetilde{G}$, then $\pi$ occurs in the correspondence for the dual pair and corresponds to a unitary representation of $\widetilde{G}^{\prime}$.

Theorem 19 [Li 1989a]. Let $G^{\prime}$ be a reductive group which is a member of some Type I reductive dual pairs. Let $\pi^{\prime}$ be a unitary irreducible genuine representation of $\widetilde{G}^{\prime}$, of low rank (in the sense of [Howe 1982]). Then there exist a unitary character $\xi$ of $\widetilde{G}^{\prime}$, a reductive group $G$ such that $\left(G, G^{\prime}\right)$ is a dual pair in the stable range with $G$ the smaller member, and a unitary genuine representation $\pi$ of $\widetilde{G}$ such that $\pi$ corresponds to $\pi^{\prime} \otimes \xi$.

As explained in [Pantano et al. 2010, Section 8.1], the correspondence for dual pairs of the form $(\operatorname{Sp}(2 n, \mathbb{R}), O(r, s))$ with $r+s$ odd can be regarded as a correspondence between genuine irreducible representations of $\operatorname{Mp}(2 n)$ and irreducible representations of $O(r, s)$. This depends on some choices, which we make the same way as we did in [Pantano et al. 2010].

For principal series representations of $O(m+1, m)$, we use the following notation. For each pair of nonnegative integers $(p, q)$ such that $p+q=m$, we write $I_{O(m+1, m)}\left(\delta^{p, q}, v\right)$ for the principal series representation of $O(m+1, m)$ with lowest $(O(m+1) \times O(m))$-type

$$
(0, \ldots, 0 ;+) \otimes(\underbrace{1, \ldots, 1}_{j}, 0, \ldots, 0 ; \epsilon)
$$

with $(j, \epsilon)=(q,+)$ if $p \geq q$, and $(p,-)$ if $p<q$. The corresponding Langlands subquotient will be denoted $J_{O(m+1, m)}\left(\delta^{p, q}, v\right)$. We say that a parameter $v$ belongs to the complementary series $\operatorname{CS}\left(O(m+1, m), \delta^{p, q}\right)$ if $J_{O(m+1, m)}\left(\delta^{p, q}, v\right)$ is unitarizable (as a representation of $O(m+1, m)$ or $\mathrm{SO}(m+1, m)$ ).

For each positive integer $m$, write $\rho_{m}$ for the infinitesimal character of the trivial representation of $\operatorname{SO}(2 m+1)$. If $v \in \mathbb{C}^{n}$, then $\left(\rho_{m} \mid v\right)$ denotes the $(m+n)$-tuple obtained by tacking the coordinates of $v$ onto $\rho_{m}$ : 


$$
\left(\rho_{m} \mid v\right)=\left(m-\frac{1}{2}, m-\frac{3}{2}, \ldots, \frac{3}{2}, \frac{1}{2}, v_{1}, \ldots, v_{n}\right) .
$$

For dual pairs of the form under consideration, the theta correspondence gives rise to the following correspondence of infinitesimal characters.

Theorem 20 [Przebinda 1996]. Let $\left(G, G^{\prime}\right)$ be a reductive dual pair with $G$ an odd orthogonal group, and $G^{\prime}$ a symplectic group, or vice versa. Let $\pi$ and $\pi^{\prime}$ be representations of $\widetilde{G}$ and $\widetilde{G}^{\prime}$, with infinitesimal characters $\gamma$ and $\gamma^{\prime}$, respectively, which correspond to each other. Assume that the rank $r$ of $G$ is greater than or equal to the rank $r^{\prime}$ of $G^{\prime}$. Then $\gamma=\left(\rho_{r-r^{\prime}} \mid \gamma^{\prime}\right)$.

We need two more results about the correspondence for symplectic-orthogonal pairs.

Theorem 21 [Adams and Barbasch 1998]. Let $n \leq m$, and write $m=n+k$. Let $p+$ $q=n$, and let $v=\left(v^{p} \mid v^{q}\right) \in \mathbb{R}^{n}$. Let $\left(G(m), G^{\prime}(n)\right)=(\operatorname{Sp}(2 m, \mathbb{R}), O(n+1, n))$ or $(O(m+1, m), \operatorname{Sp}(2 n, \mathbb{R}))$. In the correspondence for the dual pair $\left(G(m), G^{\prime}(n)\right)$,

$$
J_{G(m)}\left(\delta^{p+k, q},\left(\rho_{k} \mid v\right)\right) \longleftrightarrow J_{G^{\prime}(n)}\left(\delta^{p, q}, v\right) .
$$

Proposition 22 [Adams and Barbasch 1998]. Suppose that $\pi$ and $\pi^{\prime}$ map to each other in the theta correspondence for the dual pair $\left(G, G^{\prime}\right)=(\operatorname{Sp}(2 n, \mathbb{R}), O(r, s))$ with $r+s$ odd. If $2 n>r+s$, then the lowest $\widetilde{U}(n)$-types of $\pi$ are of minimal degree in $\pi$. Similarly, if $2 n<r+s$, then the lowest $(O(r) \times O(s))$-types of $\pi^{\prime}$ are of minimal degree in $\pi^{\prime}$.

Instead of recalling Howe's definition of the rank of a representation (see [Howe 1982]), we give a theorem which leads to an alternative definition of "low rank".

Theorem 23 [Li 1989b; 1997]. Let $G$ be $\operatorname{Mp}(2 n)$ or $O(n+1, n)$, and let $\pi$ be an irreducible unitary representation of $G$. Set

$$
r_{G}= \begin{cases}n & \text { if } G=\mathrm{Mp}(2 n) \text { or if } G=O(n+1, n) \text { with } n \text { even, } \\ n-1 & \text { if } G=O(n+1, n) \text { with } n \text { odd. }\end{cases}
$$

Let $W F(\pi) \subseteq \mathfrak{g}_{0}^{*} \simeq \mathfrak{g}_{0}$ denote the wave front set of $\pi$, and write $\operatorname{rank}(W F(\pi))$ for the maximal rank of the elements of $W F(\pi)$ as matrices. Then $\pi$ is of "low rank" in the sense of Howe if and only if

$$
\operatorname{rank}(W F(\pi))<r_{G} .
$$

Proposition 24. Let $(p, q)$ be a pair of nonnegative integers such that $p+q=n$, and let $k$ be any integer satisfying $k \geq n+2$.

(1) For all parameters $v=\left(v^{p} \mid v^{q}\right)$, if

$$
\left(\left(\rho_{k} \mid v^{p}\right) \mid v^{q}\right) \in \operatorname{CS}\left(\operatorname{SO}(n+k+1, n+k), \delta^{p+k, q}\right),
$$

then $J_{O(n+k+1, n+k)}\left(\delta^{p+k, q},\left(\left(\rho_{k} \mid \nu^{p}\right) \mid \nu^{q}\right)\right)$ is of low rank. 
(2) For all parameters $v=\left(v^{p} \mid v^{q}\right)$, if

$$
\left(\left(\rho_{k} \mid v^{p}\right) \mid v^{q}\right) \in \operatorname{CS}\left(\operatorname{Mp}(2(n+k)), \delta^{p+k, q}\right),
$$

then $J_{\mathrm{Mp}(2(n+k))}\left(\delta^{p+k, q},\left(\left(\rho_{k} \mid v^{p}\right) \mid v^{q}\right)\right)$ is of low rank.

Proof. For part (1), note that for $q=0$ this result already appears in [Adams et al. 2007, Fact 2, Section 14]; the same argument applied there goes over word for word if $q>0$. The first step is to realize $J_{O(n+k+1, n+k)}\left(\delta^{p+k, q},\left(\left(\rho_{k} \mid v^{p}\right) \mid v^{q}\right)\right)$ as a composition factor of an induced representation

$$
\operatorname{Ind}_{L}^{O(n+k+1, n+k)}(\operatorname{triv} \otimes \xi),
$$

where $L=O(k+1, k) \times \mathrm{GL}(1, \mathbb{R})^{n}$, and $\xi$ is a one-dimensional character of $\operatorname{GL}(1, \mathbb{R})^{n}$. The wave front set is then contained in the closure of the Richardson orbit for $L$, hence its rank is bounded above by the rank of that orbit. The same calculation done in [Adams et al. 2007] shows that this rank is strictly less than $n+k-1$. By Theorem 23, the representation $J_{O(n+k+1, n+k)}\left(\delta^{p+k, q},\left(\left(\rho_{k} \mid v^{p}\right) \mid v^{q}\right)\right)$ of $O(n+k+1, n+k)$ is of low rank.

For part (2), Theorem 21 implies that the Langlands quotient $J_{\mathrm{Mp}(2(n+k))}\left(\delta^{p+k, q},\left(\left(\rho_{k} \mid v^{p}\right) \mid v^{q}\right)\right)$ corresponds to $J_{O(n+1, n)}\left(\delta^{p, q}, v\right)$ in the correspondence for the pair $(\operatorname{Sp}(2(n+k), \mathbb{R}), O(n+1, n))$. By [Li 1989b, Proposition 1], if $\pi$ is any representation of $\mathrm{Mp}(2(n+k))$ coming from the duality correspondence with some representation of $O(n+1, n)$, then the rank of the wave front set of $\pi$ is at most $2 n+1$. In particular,

$$
\operatorname{rank}\left(W F\left(J_{\mathrm{Mp}(2(n+k))}\left(\delta^{p+k, q},\left(\left(\rho_{k} \mid v^{p}\right) \mid v^{q}\right)\right)\right)\right) \leq 2 n+1 .
$$

If $k>n+1$ (as in our assumptions), then this is strictly less than the split rank of $\operatorname{Mp}(2(n+k))$, hence the representation $J_{\mathrm{Mp}(2(n+k))}\left(\delta^{p+k, q},\left(\rho_{k} \mid v\right)\right)$ of $\mathrm{Mp}(2(n+$ $k)$ ) is of low rank by Theorem 23 .

One consequence of Conjecture 1 would be that the $(p, q)$-complementary series of $\mathrm{SO}(n+1, n)$ and the genuine $(p, q)$-complementary series of $\mathrm{Mp}(2 n)$ coincide. We would like to show that if $v$ is a unitary parameter for one of the groups (for a given choice of $p$ and $q$ ), then it is unitary for the other group as well. The theta correspondence provides such an argument if we know that a closely related parameter $v^{\prime}$ for a larger group of the same type is also unitary.

Theorem 25. (1) Let $v=\left(v^{p} \mid v^{q}\right) \in \operatorname{CS}\left(\operatorname{SO}(n+1, n), \delta^{p, q}\right)$. If $\left(\rho_{n+2} \mid v\right)$ is in $\mathrm{CS}\left(\mathrm{SO}(2 n+3,2 n+2), \delta^{p+n+2, q}\right)$ then $v \in \operatorname{CS}\left(\operatorname{Mp}(2 n), \delta^{p, q}\right)$.

(2) Let $v=\left(v^{p} \mid v^{q}\right) \in \operatorname{CS}\left(\operatorname{Mp}(2 n), \delta^{p, q}\right)$. If

$$
\left(\rho_{n+2} \mid v\right) \in \operatorname{CS}\left(\operatorname{Mp}(4 n+4), \delta^{p+n+2, q}\right)
$$

then $v \in \operatorname{CS}\left(\operatorname{SO}(n+1, n), \delta^{p, q}\right)$. 
Proof. For part (1), assume $v=\left(v^{p} \mid v^{q}\right) \in \mathrm{CS}\left(\mathrm{SO}(n+1, n), \delta^{p, q}\right)$, and $\left(\rho_{n+2} \mid v\right)$ is in $\operatorname{CS}\left(\operatorname{SO}(2 n+3,2 n+2), \delta^{p+n+2, q}\right)$. We want to show that $J_{\operatorname{Mp}(2 n)}\left(\delta^{p, q}, v\right)$ is unitary. By our assumption, $\pi_{s}=J_{O(2 n+3,2 n+2)}\left(\delta^{p+n+2, q},\left(\rho_{n+2} \mid v\right)\right)$ is unitary. By Proposition $24(1), \pi_{s}$ is of low rank. Theorem 19 implies that there are a character $\xi$ of $O(2 n+3,2 n+2)$, a group $\mathrm{Mp}(2 m)$ with $m \leq n+1$, and a unitary representation $\pi$ of $\mathrm{Mp}(2 m)$ such that $\pi \leftrightarrow \pi_{s} \otimes \xi$ in the theta correspondence for the dual pair $(\operatorname{Sp}(2 m, \mathbb{R}), O(2 n+3,2 n+2))$. By Proposition 22, the lowest $K$-type of $\pi_{s} \otimes \xi$ is of minimal degree for the dual pair, and must therefore occur in $\mathscr{H}$. By the explicit correspondence in $\mathscr{H}$ (see [Adams and Barbasch 1998, Proposition 2.1]), $\xi$ must be trivial. If we can show that $m=n$ then we are done, since by Theorem $21, \pi_{s}$ corresponds to $J_{\mathrm{Mp}(2 n)}\left(\delta^{p, q}, v\right)$ in the correspondence for the dual pair $(\operatorname{Sp}(2 n, \mathbb{R}), O(2 n+3,2 n+2))$, so that $J_{\mathrm{Mp}(2 n)}\left(\delta^{p, q}, v\right)$ must be unitary.

So suppose $m>n$. Then $m=n+1$. Theorem 21 tells us that $\pi_{s}$ corresponds to $J_{\mathrm{Mp}(2 n+2)}\left(\delta^{p+1, q}, v^{\prime}\right)$ for this dual pair, where $v^{\prime}=\left(n+\frac{3}{2}, v_{1}, \ldots, v_{n}\right)$ (since it is obtained from $\left(\rho_{n+2} \mid v\right)$ by removing the coordinates of $\left.\rho_{n+1}\right)$. By [Pantano et al. 2010, Proposition 7.7] (the analog, for the metaplectic group, of Proposition 10), this is not a unitary parameter for any principal series of $\operatorname{Mp}(2 n+2)$. So $m \leq n$.

Now suppose that $m<n$, say $n=m+k$. By Theorem 20, the infinitesimal character of $\pi$ is obtained from $\left(\rho_{n+2} \mid v\right)$ by removing the coordinates of $\rho_{2 n+2-m}=$ $\rho_{n+2+k}$. If $k>0$ then this means that $v$ contains a coordinate $n+\frac{5}{2}$. This implies that one of the conditions of Proposition 10 holds, hence $v$ is not a unitary parameter. This contradicts our assumption, so we must have $m=n$, and so $\pi=J_{\operatorname{Mp}(2 n)}\left(\delta^{p, q}, v\right)$ is unitary.

For part (2), let $v=\left(v^{p} \mid v^{q}\right) \in \operatorname{CS}\left(\operatorname{Mp}(2 n), \delta^{p, q}\right)$, and assume $\left(\rho_{n+2} \mid v\right)$ is in $\operatorname{CS}\left(\operatorname{Mp}(4 n+4), \delta^{p+n+2, q}\right)$. We want to show that $J_{O(n+1, n)}\left(\delta^{p, q}, v\right)$, and hence $J_{\mathrm{SO}(n+1, n)}\left(\delta^{p, q}, v\right)$, is unitary. By our assumption,

$$
\pi_{s}=J_{\mathrm{Mp}(4 n+4)}\left(\delta^{n+p+2, q},\left(\rho_{n+2} \mid \nu\right)\right)
$$

is unitary. By Proposition 24 (2), $\pi_{s}$ is of low rank. Theorem 19 implies that there are a character $\xi$ of $\mathrm{Mp}(4 n+4)$, a group $O(r, s)$ with $r+s \leq 2 n+2$ and $r+s$ odd, and a unitary representation $\pi$ of $O(r, s)$ such that $\pi_{s} \otimes \xi \leftrightarrow \pi$ in the correspondence for the pair $(\operatorname{Sp}(4 n+4, \mathbb{R}), O(r, s))$. The group $\operatorname{Mp}(4 n+4)$ has no nontrivial characters, so $\xi$ must be trivial. Also, by Proposition 22, the fine $K$-type of $\pi_{s}$ occurs in $\mathscr{H}$. By the correspondence in $\mathscr{H}$, this can only happen when $r=s+1$. We want to show that $s=n$; then $\pi=J_{O(n+1, n)}\left(\delta^{p, q}, v\right)$ is unitary.

The condition $r+s \leq 2 n+2$ implies $2 s+1 \leq 2 n+2$. By integrality, $s \leq n$. Assume $s<n$. By Theorem 20 , the infinitesimal character of $\pi$ is obtained from $\left(\rho_{n+2} \mid v\right)$ by removing the coordinates of $\rho_{2 n+2-s}$. If $s<n$ then this contains a coordinate $n+\frac{5}{2}$, which must come from $v$. Then $v$ satisfies one of the conditions of [Pantano 
et al. 2010, Proposition 7.7], hence is not a unitary parameter. Consequently $s=n$, and the theorem is proved.

The following is a direct consequence of the description of the spherical unitary parameters for the split groups of type $B$ (see Section 8).

Proposition 26 [Barbasch 2010]. If $v \in \mathrm{CS}\left(\mathrm{SO}(n+1, n), \delta_{0}\right)$, then

$$
\left(\rho_{m} \mid v\right) \in \operatorname{CS}\left(\operatorname{SO}(n+m+1, n+m), \delta_{0}\right) \text { for all } m>0 .
$$

Using this observation, Theorem 25 now immediately implies Theorem 4.

\section{Unipotent representations}

In this section, we identify the principal series parameters which are attached to special unipotent representations of $\mathrm{SO}(n+1, n)$, and discuss the unitarity of these modules.

Barbasch [2010] attaches parameters of spherical principal series representations of $\mathrm{SO}(n+1, n)_{0}$ to nilpotent orbits in $\mathfrak{s p}(2 n, \mathbb{C})$. Fix a Cartan subalgebra $\mathfrak{h}^{\vee}$ of $\mathfrak{s p}(2 n, \mathbb{C})$. This algebra is naturally isomorphic to $\mathfrak{a}^{*}$. Given a nilpotent orbit $\mathbb{O}^{\vee}$, let $\left\{e^{\vee}, h^{\vee}, f^{\vee}\right\}$ be an $\mathfrak{s l}_{2}$ triple with $f^{\vee} \in \mathcal{O}^{\vee}$ and $h^{\vee} \in \mathfrak{h}^{\vee}$. Then the corresponding spherical parameters are of the form

$$
v=\frac{h^{\vee}}{2}+\gamma,
$$

where $\gamma \in \mathfrak{z}\left(\left\{e^{\vee}, h^{\vee}, f^{\vee}\right\}\right)$, the centralizer of the triple. A spherical parameter $v$ is called unipotent if it is of the form $v=h^{\vee} / 2$ (see Definition 34). Since our $(p, q)$-principal series parameters are given by pairs of spherical parameters (for $\mathrm{SO}(p+1, p)_{0}$ and $\left.\mathrm{SO}(q+1, q)_{0}\right)$, we can attach them to pairs of nilpotent orbits analogously.

Definition 27. Fix nonnegative integers $p$ and $q$ such that $p+q=n$. A parameter $v=\left(v^{p} \mid v^{q}\right)$ for a $(p, q)$-principal series of $\mathrm{SO}(n+1, n)($ or $\mathrm{Mp}(2 n))$ is called unipotent if both $v^{p}$ and $v^{q}$ are spherical unipotent parameters for $\mathrm{SO}(p+1, p)_{0}$ and $\mathrm{SO}(q+1, q)_{0}$, respectively.

Proposition 28. Given $p$ and $q$ such that $p+q=n$, a parameter $v=\left(v^{p} \mid v^{q}\right)$ is unipotent if and only if $J\left(\delta^{p, q}, v\right)$ is a special unipotent representation of $\mathrm{SO}(n+1, n)$.

Proof. Recall first that a Langlands parameter [Langlands 1970] for $G=\mathrm{SO}(n+1, n)$ is a conjugacy class by $\operatorname{Sp}(2 n, \mathbb{C})$ of homomorphisms

$$
\varphi: W_{\mathbb{R}} \longrightarrow \operatorname{Sp}(2 n, \mathbb{C}) \times \Gamma,
$$


satisfying certain conditions. Here $\Gamma=\{\gamma, 1\}$ is the Galois group of $\mathbb{C}$ over $\mathbb{R}$, and $W_{\mathbb{R}}$ is the Weil group of $\mathbb{R}$, the group generated by $\mathbb{C}^{\times}$and an element $\tau$, subject to the relations $\tau z \tau^{-1}=\bar{z}$ for $z \in \mathbb{C}^{\times}, \tau^{2}=-1$. To each such parameter $\varphi$ is associated an " $L$-packet", which is a finite set $\Pi_{\varphi}$ of irreducible admissible representations of $G$. The assignment is explained in detail in [Borel 1979].

For principal series representations of $\mathrm{SO}(n+1, n)$, the $L$-packets are in fact singletons. Fix a maximal torus $T$ of $\operatorname{Sp}(2 n, \mathbb{C})$, and $\pi=J\left(\delta^{p, q}, v\right)$. Then the Langlands parameter $\varphi$ of $\pi$ can be chosen to be

$$
\begin{gathered}
\varphi(\tau)=((\underbrace{1, \ldots, 1}_{p}, \underbrace{-1, \ldots,-1}_{q}), \gamma) \in T \times \Gamma ; \\
\varphi\left(r e^{i \theta}\right)=r^{2 v}=\left(\left(r^{2 v_{1}}, \ldots, r^{2 v_{n}}\right), 1\right) .
\end{gathered}
$$

Now recall from [Arthur 1989, §4] (see also [Adams et al. 1992, Chapters 22 and 26]) that an Arthur parameter for $G=\mathrm{SO}(n+1, n)$ is a conjugacy class by $\operatorname{Sp}(2 n, \mathbb{C})$ of certain maps

$$
\psi: W_{\mathbb{R}} \times \operatorname{SL}(2, \mathbb{C}) \longrightarrow \operatorname{Sp}(2 n, \mathbb{C}) \times \Gamma,
$$

whose restriction to $\operatorname{SL}(2, \mathbb{C})$ must be a holomorphic homomorphism into $\operatorname{Sp}(2 n, \mathbb{C})$. To each Arthur parameter is attached a finite set of irreducible admissible representations, called an Arthur packet. If $\psi$ is an Arthur parameter, then

$$
\varphi_{\psi}: W_{\mathbb{R}} \longrightarrow \operatorname{Sp}(2 n, \mathbb{C}) \times \Gamma,
$$

defined by

$$
\varphi_{\psi}(w)=\psi\left(w,\left(\begin{array}{cc}
|w|^{\frac{1}{2}} & 0 \\
0 & |w|^{-\frac{1}{2}}
\end{array}\right)\right)
$$

for all $w \in W_{\mathbb{R}}$, is a Langlands parameter. Here, if $w=z \tau$ then $|w|=|z|$.

An Arthur parameter $\psi$ is called unipotent if

$$
\psi\left(\mathbb{C}^{\times}\right)=\{(1,1)\} .
$$

We call representations which are contained in an $L$-packet $\Pi_{\varphi_{\psi}}$ for $\psi$ a unipotent parameter special unipotent. If the principal series representation $\pi=J\left(\delta^{p, q}, v\right)$ is special unipotent, then the corresponding Arthur parameter $\psi$ must satisfy (7-4) and (7-1a), and the image of $\operatorname{SL}(2, \mathbb{C})$ must lie in the centralizer $C$ of $\psi(\tau)$. This centralizer is isomorphic to $\operatorname{Sp}(2 p, \mathbb{C}) \times \operatorname{Sp}(2 q, \mathbb{C})$. It follows that our parameters are in 1-1 correspondence with $C$-orbits of holomorphic maps of $\mathrm{SL}(2, \mathbb{C})$ into $C$. The nontrivial orbits are given by $C$-conjugacy classes of embeddings of $\mathfrak{s l}(2, \mathbb{C})$ into $\mathfrak{s p}(2 p, \mathbb{C}) \oplus \mathfrak{s p}(2 q, \mathbb{C})$, and such classes of embeddings are in turn in 1-1 correspondence with nonzero nilpotent $C$-orbits on $\mathfrak{s p}(2 p, \mathbb{C}) \oplus \mathfrak{s p}(2 q, \mathbb{C})$, so that the set of Arthur parameters under consideration is indeed in 1-1 correspondence 
with pairs of nilpotent orbits as claimed. Unwinding the definitions, we see that given such a pair of orbits $\left(\mathrm{O}_{p}^{\vee}, \mathrm{O}_{q}^{\vee}\right)$, the corresponding Arthur parameter $\psi$ determines a Langlands parameter $\varphi_{\psi}$ which is the parameter of a $(p, q)$-principal series. Moreover

$$
d \psi\left(\left(\begin{array}{cc}
\frac{1}{2} & 0 \\
0 & -\frac{1}{2}
\end{array}\right)\right)
$$

which, in the case of principal series, is the continuous parameter of the representations attached to $\varphi_{\psi}$, is then $\left(h_{p}^{\vee} / 2, h_{q}^{\vee} / 2\right)$, where $h_{p}^{\vee}$ and $h_{q}^{\vee}$ are the middle elements of $\mathfrak{s l}_{2}$ triples for $\mathbb{O}_{p}^{\vee}$ and $\mathcal{O}_{q}^{\vee}$, respectively. This completes the proof of our proposition.

In his recent book, Arthur [2013] reformulates and proves several of the conjectures of [Arthur 1989]. In particular, for certain quasisplit classical groups including $\mathrm{SO}(n+1, n)$, he proves that all representations in certain Arthur packets are local components of automorphic representations, and therefore unitary (see Theorem 1.5). These include the Arthur packets attached to unipotent Arthur parameters. Moreover he proves (see Proposition 7.4.1) that these Arthur packets contain the $L$-packets that are attached to them. Consequently, we obtain the following result.

Theorem 29 [Arthur 2013]. Special unipotent representations of $\mathrm{SO}(n+1, n)$ are unitary.

Theorem 29, Proposition 28, part (1) of Theorem 25, and the observation that if $v$ is a unipotent parameter, then so is $\left(\rho_{n+2} \mid v\right)$, now easily imply Theorem 6 .

\section{The spherical unitary dual of $\operatorname{SO}(n+1, n)_{0}$.}

In this section, we give an explicit description of the spherical unitary dual of split groups of type $B$. All the results are known, and due to D. Barbasch [2010; 2008]. See also [Pantano et al. 2010, Section 11] for a more detailed account.

Let $G=\mathrm{SO}(n+1, n)_{0}$, and let $\check{\mathfrak{g}}=\mathfrak{s p}(2 n, \mathbb{C})$ be the complex dual Lie algebra, with Cartan subalgebra $\mathfrak{h}$. The spherical unitary dual of $G$ is a disjoint union of sets, parametrized by nilpotent orbits in $\mathfrak{\mathfrak { g }}$. Recall that nilpotent orbits in $\mathfrak{s p}(2 n, \mathbb{C})$ are parametrized by partitions of $2 n$ in which every odd part occurs with even multiplicity.

Definition 30. Let $v$ be a real parameter in $\check{\mathfrak{h}}$, and let $\mathcal{O}$ be a nilpotent orbit in $\check{\mathfrak{g}}$. Let $\breve{h} \in \mathfrak{h}$ be the middle element of an $\mathfrak{s l}(2)$ triple associated to $\mathcal{O}$. We say that $v$ is attached to $\mathbb{O}$ if

(1) $v=\check{h} / 2+\kappa$, for some semisimple element $\kappa$ in the centralizer $\mathfrak{Z}_{\breve{\mathfrak{g}}}(\mathcal{O})$, and

(2) whenever $\mathscr{O}^{\prime}$ is another nilpotent orbit in $\check{\mathfrak{g}}$ such that $v=\check{h}^{\prime} / 2+\kappa^{\prime}$, for some $\kappa^{\prime} \in \mathfrak{Z}_{\mathfrak{g}}\left(\breve{O}^{\prime}\right)$ semisimple, then $\widetilde{O}^{\prime} \subset \overline{\mathcal{O}}$. 
If $v$ is a real parameter in $\check{\mathfrak{h}}$, we can identify $v$ with an element of $\mathfrak{a}_{\mathbb{R}}^{*}$, and consider the irreducible spherical representation $J\left(\delta_{0}, v\right)$ of $G$.

Definition 31. A parameter $v \in \mathfrak{a}_{\mathbb{R}}^{*}$ is in the $\mathbb{O}$-complementary series if

(1) $v$ is attached to 0 , and

(2) $J\left(\delta_{0}, v\right)$ is unitary.

The zero-complementary series, that is, the complementary series attached to the trivial nilpotent orbit plays a special role.

Theorem 32 [Barbasch 2010]. For every nilpotent orbit $\mathbb{O}$ in $\mathfrak{\mathfrak { g }}$, let $G^{0}(\mathcal{O})$ be the connected real split group whose complex dual Lie algebra is $\mathfrak{Z}_{\mathfrak{g}}(0)$. Let $v=\breve{h} / 2+\kappa$ be a parameter attached to the nilpotent orbit $\mathrm{O}$. Then $v$ is in the $\mathrm{O}$-complementary series for the group $G$ if and only if $\kappa$ is in the zero-complementary series for the group $G^{0}(0)$.

The zero-complementary series of all real split groups is known, thanks to D. Barbasch. We recall the result for the groups we need.

Theorem 33 [Barbasch 2010]. The zero-complementary series for split groups of type $B_{k}, C_{k}$ and $D_{k}$ consists of the following dominant parameters:

$B_{k}$. The set of all $v=\left(\kappa_{1}, \ldots, \kappa_{k}\right)$ such that $0 \leq \kappa_{1} \leq \kappa_{2} \leq \cdots \leq \kappa_{k}<\frac{1}{2}$.

$C_{k}$. The set of all $v=\left(\kappa_{1}, \ldots, \kappa_{k}\right)$ such that there exists an index $i=2, \ldots, k$ with the property that

$$
0 \leq \kappa_{1} \leq \cdots \leq \kappa_{i}<1-\kappa_{i-1}<\kappa_{i+1}<\cdots<\kappa_{k}<1,
$$

and, for every $i \leq j<k$, there is an odd number of $\kappa_{l}$ with $1 \leq l<i$ such that $\kappa_{j}<1-\kappa_{l}<\kappa_{j+1}$.

$D_{k}$. Similar to type $C_{k}$. If $k$ is even, replace every occurrence of $\kappa_{1}$ by $\left|\kappa_{1}\right|$. If $k$ is odd, replace every occurrence of $\kappa_{1}$ by 0.

Note that the choice of dominant parameters is not the standard one.

To compute $\mathfrak{Z}_{\mathfrak{g}}(\mathcal{O})$, let $\lambda$ be the partition corresponding to $\mathbb{O}$; denote the parts of $\lambda$ by $a_{l}$, and their multiplicity by $r_{l}$ ( $r_{l}$ is even if $a_{l}$ is odd):

$$
\lambda=(\underbrace{a_{1}, \ldots, a_{1}}_{r_{1}}, \ldots, \underbrace{a_{m}, \ldots, a_{m}}_{r_{m}}) .
$$

Then $\mathfrak{Z}_{\mathfrak{g}}(\mathbb{O})$ is a product of symplectic and orthogonal Lie algebras. There is a factor $\mathfrak{s p}\left(r_{l}\right)$ for each odd part, and a factor $\mathfrak{s o}\left(r_{l}\right)$ for each even part.

We describe the contribution of an odd part $a$ of $\lambda$ to $v$; we refer the reader to the appendix of [Pantano et al. 2010] for the other cases. If $r_{a}=2 n_{a}$, the partition $\lambda$ 
contains $n_{a}$ pairs of the form $(a, a)$. The $j$-th pair $(a, a)$ contributes a string

$$
-\left(\frac{a-1}{2}\right),-\left(\frac{a-3}{2}\right), \ldots,-1,0,+1, \ldots,+\left(\frac{a-3}{2}\right),+\left(\frac{a-1}{2}\right)
$$

(of length $a$ ) to $\check{h} / 2$, and a string $\left(\kappa_{j}^{(a)}, \kappa_{j}^{(a)}, \ldots, \kappa_{j}^{(a)}\right.$ ) (also of length $a$ ) to $\kappa$. Moreover the part $a$ contributes a factor $\mathfrak{s p}\left(2 n_{a}\right)$ to the stabilizer of the orbit, and a factor $\mathrm{SO}\left(n_{a}+1, n_{a}\right)_{0}$ to the group $G^{0}(\tilde{O})$. For $v$ to be a unitary parameter, we impose the condition that the parameter $\left(\kappa_{1}^{(a)}, \ldots, \kappa_{n_{a}}^{(a)}\right)$ belongs to the zerocomplementary series for $\mathrm{SO}\left(n_{a}+1, n_{a}\right)_{0}$ (see Theorem 33).

Definition 34 (Barbasch). A parameter $v=\check{h} / 2+\kappa$ is called spherical unipotent if $\kappa=0$.

Finally we give an explicit list of the spherical unitary parameters (in the fundamental Weyl chamber, FWC) for $\mathrm{SO}(n+1, n)_{0}$ with $n \leq 3$.

(1) For $n=1$, the closed interval $\left[0, \frac{1}{2}\right]$.

(2) For $n=2$,

- The intersection of the unit cube with the FWC: $\left\{0 \leq v_{2} \leq v_{1} \leq \frac{1}{2}\right\}$,

- The isolated point $\left(\frac{3}{2}, \frac{1}{2}\right)$.

(3) For $n=3$,

- The intersection of the unit cube with the FWC: $\left\{0 \leq v_{3} \leq v_{2} \leq v_{1} \leq \frac{1}{2}\right\}$.

- The segment from $\left(\frac{1}{2}, \frac{1}{2}, \frac{1}{2}\right)$ to $\left(1, \frac{1}{2}, 0\right)$ :

$$
\left\{\left(\frac{1}{2}+t, \frac{1}{2}, \frac{1}{2}-t\right), \text { for } 0 \leq t \leq \frac{1}{2}\right\} .
$$

- The segment from $\left(1, \frac{1}{2}, 0\right)$ to $\left(\frac{3}{2}, \frac{1}{2}, \frac{1}{2}\right)$ :

$$
\left\{\left(1+t, \frac{1}{2}, t\right) \text {, for } 0 \leq t \leq \frac{1}{2}\right\} .
$$

- The segment from $(1,1,0)$ to $\left(\frac{3}{2}, \frac{1}{2}, \frac{1}{2}\right)$ :

$$
\left\{(1+t, 1-t, t) \text {, for } 0 \leq t \leq \frac{1}{2}\right\} .
$$

- The segment from $\left(\frac{3}{2}, \frac{1}{2}, 0\right)$ to $\left(\frac{3}{2}, \frac{1}{2}, \frac{1}{2}\right)$ :

$$
\left\{\left(\frac{3}{2}, \frac{1}{2}, t\right) \text {, for } 0 \leq t \leq \frac{1}{2}\right\} \text {. }
$$

- The isolated point $\left(\frac{5}{2}, \frac{3}{2}, \frac{1}{2}\right)$.

\section{Acknowledgements}

We thank Jeffrey Adams for explaining Arthur's results on unipotent representations and their relevance to our work. Moreover we are grateful to the referee for several valuable suggestions. 


\section{References}

[Adams and Barbasch 1998] J. Adams and D. Barbasch, "Genuine representations of the metaplectic group”, Compositio Math. 113:1 (1998), 23-66. MR 99h:22013 Zbl 0913.11022

[Adams et al. 1992] J. Adams, D. Barbasch, and D. A. Vogan, Jr., The Langlands classification and irreducible characters for real reductive groups, Progress in Mathematics 104, Birkhäuser, Boston, 1992. MR 93j:22001 Zbl 0756.22004

[Adams et al. 2007] J. Adams, D. Barbasch, A. Paul, P. E. Trapa, and D. A. Vogan, Jr., "Unitary Shimura correspondences for split real groups", J. Amer. Math. Soc. 20:3 (2007), 701-751. MR 2008i:22008 Zbl 1114.22009

[Arthur 1989] J. G. Arthur, "Unipotent automorphic representations: conjectures", pp. 13-71 in Orbites unipotentes et représentations, II: Groupes p-adiques et réels, edited by M. Andler, Astérisque 171-172, Société Mathématique de France, Paris, 1989. MR 91f:22030 Zbl 0728.22014

[Arthur 2013] J. G. Arthur, The endoscopic classification of representations: orthogonal and symplectic groups, American Mathematical Society Colloquium Publications 61, American Mathematical Society, Providence, RI, 2013. MR 3135650 Zbl 06231010

[Barbasch 2004] D. Barbasch, "Relevant and petite $K$-types for split groups", pp. 35-71 in Functional analysis VIII (Dubrovnik, 2003), edited by D. Bakić et al., Various Publ. Ser. (Aarhus) 47, Aarhus University, Aarhus, 2004. MR 2006d:22020 Zbl 1070.22006

[Barbasch 2010] D. Barbasch, "The unitary spherical spectrum for split classical groups", J. Inst. Math. Jussieu 9:2 (2010), 265-356. MR 2011d:22014 Zbl 1188.22010

[Barbasch et al. 2008] D. Barbasch, D. Ciubotaru, and A. Pantano, "Unitarizable minimal principal series of reductive groups", pp. 63-136 in Representation theory of real reductive Lie groups, edited by J. Arthur et al., Contemp. Math. 472, American Mathematical Society, Providence, RI, 2008. MR 2011e:22020 Zbl 1178.22017

[Borel 1979] A. Borel, "Automorphic L-functions", pp. 27-61 in Automorphic forms, representations and L-functions, Part 2 (Corvallis, OR, 1977), edited by A. Borel and W. Casselman, Proc. Sympos. Pure Math. 33, American Mathematical Society, Providence, RI, 1979. MR 81m:10056 Zbl 0412.10017

[Fulton and Harris 1991] W. Fulton and J. Harris, Representation theory: a first course, Graduate Texts in Mathematics 129, Springer, New York, 1991. MR 93a:20069 Zbl 0744.22001

[Howe 1982] R. Howe, "On a notion of rank for unitary representations of the classical groups", pp. 224-331 in Harmonic analysis and group representation (Cortona, 1980), edited by A. Figà Talamanca, CIME Summer Schools 82, Liguori, Naples, 1982. Reprinted by Springer, Berlin, 2011. MR 86j:22016

[Howe 1989] R. Howe, “Transcending classical invariant theory", J. Amer. Math. Soc. 2:3 (1989), 535-552. MR 90k:22016 Zbl 0716.22006

[Knapp and Vogan 1995] A. W. Knapp and D. A. Vogan, Jr., Cohomological induction and unitary representations, Princeton Mathematical Series 45, Princeton University Press, 1995. MR 96c:22023 Zbl 0863.22011

[Knapp and Zuckerman 1977] A. W. Knapp and G. Zuckerman, "Classification theorems for representations of semisimple Lie groups", pp. 138-159 in Non-commutative harmonic analysis (MarseilleLuminy, 1976), edited by J. Carmona and M. Vergne, Lecture Notes in Math. 587, Springer, Berlin, 1977. MR 57 \#16474 Zbl 0353.22011

[Langlands 1970] R. P. Langlands, "Problems in the theory of automorphic forms", pp. 18-61 in Lectures in modern analysis and applications, III, edited by C. T. Taam, Lecture Notes in Math. 170, Springer, Berlin, 1970. MR 46 \#1758 Zbl 0225.14022 
[Li 1989a] J.-S. Li, "On the classification of irreducible low rank unitary representations of classical groups”, Compositio Math. 71:1 (1989), 29-48. MR 90k:22027 Zbl 0694.22012

[Li 1989b] J.-S. Li, "On the singular rank of a representation", Proc. Amer. Math. Soc. 106:2 (1989), 567-571. MR 89k:22029 Zbl 0682.22009

[Li 1989c] J.-S. Li, "Singular unitary representations of classical groups", Invent. Math. 97:2 (1989), 237-255. MR 90h:22021 Zbl 0694.22011

[Li 1997] J.-S. Li, "Unipotent representations attached to small nilpotent orbits", 1997, Available at http://www.math.umd.edu/ jda/seattle_proceedings/li.ps. Lecture notes from a conference on Representation theory of real and p-adic reductive groups, (Seattle, WA, 1997).

[Littlewood 1944] D. E. Littlewood, "On invariant theory under restricted groups", Philos. Trans. Roy. Soc. London. Ser. A. 239 (1944), 387-417. MR 7,6e Zbl 0060.04403

[Pantano et al. 2010] A. Pantano, A. Paul, and S. A. Salamanca-Riba, "Unitary genuine principal series of the metaplectic group", Represent. Theory 14 (2010), 201-248. MR 2011a:22013 Zbl 1200.22006

[Przebinda 1996] T. Przebinda, "The duality correspondence of infinitesimal characters", Colloq. Math. 70:1 (1996), 93-102. MR 96m:22034 Zbl 0854.22017

[Serre 1977] J.-P. Serre, Linear representations of finite groups, Graduate Texts in Mathematics 42 , Springer, New York, 1977. MR 56 \#8675 Zbl 0355.20006

[Speh and Vogan 1980] B. Speh and D. A. Vogan, Jr., "Reducibility of generalized principal series representations", Acta Math. 145:3-4 (1980), 227-299. MR 82c:22018 Zbl 0457.22011

[Vogan 1981] D. A. Vogan, Jr., Representations of real reductive Lie groups, Progress in Mathematics 15, Birkhäuser, Boston, 1981. MR 83c:22022 Zbl 0469.22012

[Vogan 1993] D. A. Vogan, Jr., "Unipotent representations and cohomological induction", pp. 4770 in The Penrose transform and analytic cohomology in representation theory (South Hadley, MA, 1992), edited by M. Eastwood et al., Contemp. Math. 154, American Mathematical Society, Providence, RI, 1993. MR 94j:22017 Zbl 0822.22009

Received July 7, 2013. Revised June 24, 2014.

\author{
ALESSANDRA PANTANO \\ DEPARTMENT OF MATHEMATICS \\ UNIVERSITY OF CALIFORNIA, IRVINE \\ IRVINE, CA 92697 \\ UNITED STATES \\ apantano@uci.edu
}

AnNegret Paul

DEPARTMENT OF MATHEMATICS

WESTERN MICHIGAN UNIVERSITY

KALAMAZOO, MI 49008

UNITED STATES

annegret.paul@wmich.edu

Susana SALAMANCA Riba

DEPARTMENT OF MATHEMATICS

New Mexico State University

LAS CRUCES, NM 88003

UNITED STATES

ssalaman@nmsu.edu 


\title{
PACIFIC JOURNAL OF MATHEMATICS
}

\author{
msp.org/pjm
}

Founded in 1951 by E. F. Beckenbach (1906-1982) and F. Wolf (1904-1989)

\section{EDITORS}

Don Blasius (Managing Editor)

Department of Mathematics

University of California

Los Angeles, CA 90095-1555

blasius@math.ucla.edu

\author{
Paul Balmer \\ Department of Mathematics \\ University of California \\ Los Angeles, CA 90095-1555 \\ balmer@math.ucla.edu \\ Robert Finn \\ Department of Mathematics \\ Stanford University \\ Stanford, CA 94305-2125 \\ finn@math.stanford.edu \\ Sorin Popa \\ Department of Mathematics \\ University of California \\ Los Angeles, CA 90095-1555 \\ popa@math.ucla.edu
}

\author{
Vyjayanthi Chari \\ Department of Mathematics \\ University of California \\ Riverside, CA 92521-0135 \\ chari@math.ucr.edu \\ Kefeng Liu \\ Department of Mathematics \\ University of California \\ Los Angeles, CA 90095-1555 \\ liu@math.ucla.edu \\ Jie Qing \\ Department of Mathematics \\ University of California \\ Santa Cruz, CA 95064 \\ qing@ cats.ucsc.edu
}

\section{PRODUCTION}

Silvio Levy, Scientific Editor, production@msp.org

\section{SUPPORTING INSTITUTIONS}

ACADEMIA SINICA, TAIPEI

CALIFORNIA INST. OF TECHNOLOGY

INST. DE MATEMÁTICA PURA E APLICADA

KEIO UNIVERSITY

MATH. SCIENCES RESEARCH INSTITUTE

NEW MEXICO STATE UNIV.

OREGON STATE UNIV.

\author{
STANFORD UNIVERSITY \\ UNIV. OF BRITISH COLUMBIA \\ UNIV. OF CALIFORNIA, BERKELEY \\ UNIV. OF CALIFORNIA, DAVIS \\ UNIV. OF CALIFORNIA, LOS ANGELES \\ UNIV. OF CALIFORNIA, RIVERSIDE \\ UNIV. OF CALIFORNIA, SAN DIEGO \\ UNIV. OF CALIF., SANTA BARBARA
}

\author{
Daryl Cooper \\ Department of Mathematics \\ University of California \\ Santa Barbara, CA 93106-3080 \\ cooper@math.ucsb.edu \\ Jiang-Hua Lu \\ Department of Mathematics \\ The University of Hong Kong \\ Pokfulam Rd., Hong Kong \\ jhlu@maths.hku.hk \\ Paul Yang \\ Department of Mathematics \\ Princeton University \\ Princeton NJ 08544-1000 \\ yang@math.princeton.edu
}

These supporting institutions contribute to the cost of publication of this Journal, but they are not owners or publishers and have no responsibility for its contents or policies.

See inside back cover or msp.org/pjm for submission instructions.

The subscription price for 2014 is US $\$ 410 /$ year for the electronic version, and \$535/year for print and electronic.

Subscriptions, requests for back issues and changes of subscribers address should be sent to Pacific Journal of Mathematics, P.O. Box 4163, Berkeley, CA 94704-0163, U.S.A. The Pacific Journal of Mathematics is indexed by Mathematical Reviews, Zentralblatt MATH, PASCAL CNRS Index, Referativnyi Zhurnal, Current Mathematical Publications and Web of Knowledge (Science Citation Index).

The Pacific Journal of Mathematics (ISSN 0030-8730) at the University of California, c/o Department of Mathematics, 798 Evans Hall \#3840, Berkeley, CA 94720-3840, is published twelve times a year. Periodical rate postage paid at Berkeley, CA 94704, and additional mailing offices. POSTMASTER: send address changes to Pacific Journal of Mathematics, P.O. Box 4163, Berkeley, CA 94704-0163.

PJM peer review and production are managed by EditFLOW ${ }^{\circledR}$ from Mathematical Sciences Publishers.

\section{PUBLISHED BY}

\section{mathematical sciences publishers \\ nonprofit scientific publishing}

http://msp.org/

(C) 2014 Mathematical Sciences Publishers 


\section{PACIFIC JOURNAL OF MATHEMATICS}

Volume $271 \quad$ No. $2 \quad$ October 2014

Monoids of modules and arithmetic of direct-sum decompositions

NiChOlas R. BAETH and AlFRED GEROLDINGER

On the torsion anomalous conjecture in $\mathrm{CM}$ abelian varieties

SARA CHECCOLI and EVELINA VIADA

Eigenvalue estimate and compactness for closed $f$-minimal surfaces

347

Xu Cheng, Tito Mejia and Detang Zhou

Lefschetz numbers of symplectic involutions on arithmetic groups

369

STEFFEN KIONKE

Categorification of a parabolic Hecke module via sheaves on moment 415 graphs

MARTINA LANINI

Unitary representations of $\operatorname{GL}(n, K)$ distinguished by a Galois

445 involution for a $p$-adic field $K$

NADIR MATRINGE

On $f$-biharmonic maps and $f$-biharmonic submanifolds

461

YE-LIN OU

Unitary principal series of split orthogonal groups

Alessandra Pantano, AnNegret Paul and Susana SALAMANCA RIBA 